\title{
Transport coefficients for the simple reacting spheres kinetic model I: reaction rate and shear viscosity
}

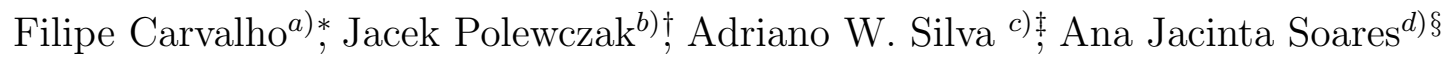 \\ ${ }^{a)}$ Polytechnic Institute of Viana do Castelo, Portugal \\ ${ }^{b)}$ Department of Mathematics, California State University, Northridge (CA), USA \\ ${ }^{c)}$ Instituto Federal de Educação, Ciência e Tecnologia do Paraná, Curitiba, Brazil \\ ${ }^{d)}$ Centro de Matemática, Universidade do Minho, Braga, Portugal \\ Accepted, Physica A, March 27, 2018
}

\begin{abstract}
In this work, we consider a dilute reactive mixture of four constituents undergoing the reversible reaction $A+B \rightleftharpoons C+D$. The mixture is described by the Simple Reacting Spheres (SRS) kinetic model which treats both elastic and reactive collisions as hard spheres type and introduces a "correction" term in the elastic operator in order to prevent double counting of the events in the collisional integrals. We use the Chapman-Enskog method, at the first-order level of the Enskog expansion, to determine the non-equilibrium solution to the SRS system in a chemical regime for which both elastic and reactive collisions occur with comparable characteristic times. We then determine the transport coefficients and focus our analysis on the evaluation of those coefficients associated with the reaction rate and the shear viscosity. Our numerical evaluation of the transport coefficients allows for the investigation of their behaviour in a suitably chosen parametric space with an opportunity to check how these coefficients are influenced by the chemical reaction and by the "correction" term proper of the SRS model.
\end{abstract}

Keywords: Boltzmann equation. Chemical reactions. Enskog expansion. Transport coefficients

PACS: 47.45.Ab, 47.70.Fw, 51.10.+y, 51.20.+d, 82.20.Pm, 82.20.Fd.

\section{Introduction}

Non-equilibrium effects and transport properties of chemically reactive mixtures are widely investigated in modern fluid dynamics, due to several applications of multicomponent reactive systems in chemical engineering, biotechnology, combustion engineering, propulsion devices and other industrial processes. The description of the chemical kinetics of the reaction mechanism is an important part of the investigation and the application of mathematical models can provide some additional tools to better understand the chemical process [1].

\footnotetext{
*filipecarvalho@esce.ipvc.pt

†jacek.polewczak@csun.edu

‡adriano.silva@ifpr.edu.br

§ajsoares@math.uminho.pt
} 
At this level, the kinetic theory of chemically reactive mixtures improves the knowledge of the reaction dynamics and provides the expressions for the reaction rates of the chemical species [2, 3, 4]. There are many contributions in this field, after the pioneering works by Prigogine, Xhrouet and Mahieu [5, 6], Present [7, 8, Ludwig and Heil [9], Ross and Mazur [10], and here we quote, for example, papers [11, 12, 13, 14, 15, 16, 17, 18, 19, 20, 21, 22, 23, 24, 25, 26, 27, 28, 29, 30] which essentially deal with reaction rates and non-equilibrium effects induced by chemical reactions, as well as transport properties and influence of the chemical reaction on transport coefficients.

Broadly speaking, the above mentioned papers use the molecular description proper of the kinetic theory of gases to deduce explicit expressions for both the reaction rates and the transport coefficients and then investigate in detail the role of the chemical reaction in different processes.

The present paper also arises in this direction, but considers a particular kinetic model for reactive mixture, namely the simple reacting spheres (SRS) kinetic model, which has been developed by Xystris and Dahler [13] and further advanced by Dahler and Qin [31, 32. In the SRS kinetic model, elastic and reactive collisions are assumed of hard-sphere type and are treated in equal par, contrary to other models that treat the reactive terms as a small perturbation of the elastic ones (see, for example, [5, 6, 11, 12]). Therefore, the SRS model results to be appropriate to investigate processes in which chemical reactions play a relevant role. This is the case of the analysis developed in the present paper.

Furthermore, the kinetic equations of the SRS model includes a "correction" term in the elastic operator in order to describe appropriately the collisional dynamics and prevent double counting of the events in the collisional integrals. In contrast to most kinetic models for chemically reactive mixtures (see, for example, [2, 20, 21, 23, 26, 27]), the SRS model has built-in the micro-reversibility principle (detailed balance) and its satisfaction can be explicitly verified. Additionally, the SRS kinetic theory, if considered in its general formulation, provides a kinetic system of equations of the revised Enskog type for reactive mixtures and this permits to extend the mathematical approach to moderate dense regimes. For the details on the SRS model, see the original papers [13, 31, 32] and also the mathematical analysis developed in papers [33, 34, 35, 36, 37.

Motivated by the robustness of the SRS model, on one side, and by the interest in the detailed description of the chemical kinetics of reactive processes, on the other side, we plan to develop a research program oriented for the explicit evaluation of the reaction rates and transport coefficients, as well as for the influence of the chemical reaction on the related properties of interest.

In this paper, we start with a simpler case of the SRS model in the dilute gas regime, before considering the more challenging case of the SRS model in dense gas regime, and focus our analysis on the evaluation of the transport coefficients associated to reaction rate and shear viscosity of the reactive mixture.

The SRS model in this work is applied to a quaternary reactive mixture of monatomic gases, whose constituents undergo the reversible reaction of bimolecular type. We use the Chapman-Enskog method and the second approximation of the Enskog expansion, to determine the non-equilibrium solution to the SRS system in a chemical regime of fast chemical reaction for which both elastic and reactive collisions occur with comparable characteristic times. We then determine the transport coefficients and investigate the influence of the chemical reaction 
on such coefficients. We also analyse the impact of the elastic correction term proper of the SRS model on the transport properties.

The paper is organized as follows. In Section 2, we briefly describe the SRS model, with emphasis on the kinetic system, equilibrium properties and macroscopic equations for the hydrodynamic fields. In Section 3, we present the transport algorithm based on the Chapman-Enskog method to obtain the second approximate solution (or the first-order solution) to the SRS kinetic equations. Then, in Section 4, we explicitly derive the linear integral equations for the determination of the transport coefficients associated to the reaction rate and shear viscosity. In Section 5, we implement numerically the transport algorithm presented in Section 3 and use the integral equations derived in Section 4 to evaluate the coefficients of the reaction rate and shear viscosity. We perform many numerical computations in a broadly varying parametric space in order to analyse the influence of the chemical reaction on the behaviour of the transport coefficients. We also investigate the impact of the elastic correction term of the SRS model on the transport coefficients. Finally, in Section 6 we summarize our results and present the final remarks.

\section{The SRS kinetic model}

In this section we briefly describe the SRS kinetic model for a dilute reactive mixture. For a detailed description, see, for example, [35, 36, 37] and the references cited therein.

We consider a four component mixture $A, B, C, D$ whose particles behave as if they were single mass points. Internal degrees of freedom for the gas particles, such as vibrational and rotational energies, are not taken into account. Particles undergo binary elastic collisions and reactive encounters according to a reversible chemical reaction of bimolecular type,

$$
A+B \rightleftharpoons C+D \text {. }
$$

We use the indices 1, 2, 3, 4 for the constituents $A, B, C, D$, respectively. Moreover, we denote by $m_{i}, d_{i}$ and $E_{i}$ the molecular mass, the molecular diameter and the chemical binding energy of each $i$-constituent, $i=1, \ldots, 4$. Reactive collisions occur when the reactive particles are separated by a distance $\sigma_{12}=\frac{1}{2}\left(d_{1}+d_{2}\right)$ or $\sigma_{34}=\frac{1}{2}\left(d_{3}+d_{4}\right)$, and result in a redistribution of masses and in a rearrangement of energies (kinetic and binding) among the constituents. In particular, the conservation of mass during chemical reactions, see (1), implies that

$$
m_{1}+m_{2}=m_{3}+m_{4}=M .
$$

Moreover a conservation law of the total energy holds during reactive collisions, and the balance of chemical binding energy of products and reactants defines the reaction heat by $E_{a b s}=E_{3}+$ $E_{4}-E_{1}-E_{2}$. Thus, $E_{a b s}>0$ means that the forward reaction $A+B \rightarrow C+D$ is endothermic and $E_{a b s}$ represents the absorbed heat energy, whereas $E_{a b s}<0$ means that the forward reaction is exothermic and $E_{a b s}$ represents the energy released in the form of heat. We also introduce $\gamma_{1}=\gamma_{2}$ and $\gamma_{3}=\gamma_{4}$ representing the activation energy of the forward and backward reactions, respectively, and we have $E_{a b s}=\gamma_{1}-\gamma_{3}$.

From here on, we use indices $i, j, k, l$ in such a way that

$$
(i, j, k, l) \in\{(1,2,3,4),(2,1,4,3),(3,4,1,2),(4,3,2,1)\}
$$


where $j, k, l$ are uniquely defined for each $i=1, \ldots, 4$, fixed, and that $j$ and $l$ are the unique partners of $i$ and $k$, respectively, in the chemical reaction (1).

\subsection{The dilute SRS kinetic system}

In the SRS kinetic model, the chemical reaction (1) is realized via binary hard-spheres elastic and reactive collisions. An elastic collision between particles from constituents $i$ and $s$ results in a change of their velocities, but with the conservation of linear momentum and kinetic energy. On the other hand, a reactive collision between particles from constituents $i$ and $j$ results in a transition of the reactants $A_{i}$ and $A_{j}$ into products $A_{k}$ and $A_{l}$, a consequent change of their velocities, and a rearrangement of masses and redistribution of the internal energies.

We point out that both elastic and reactive collisions are of hard-spheres type. In particular, reactive collisions occur if the kinetic energy associated with the relative motion along the line of centres exceeds the activation energy. We introduce coefficients $\beta_{i j}$, with $0 \leq \beta_{i j} \leq 1$, to indicate that only a fraction $\beta_{i j}$ of collisions with high energy result in a chemical reaction. Thus, coefficients $\beta_{i j}$ play the role of steric factors.

We introduce the one-particle distribution functions $f_{i}(t, x, v)$, for $i=1, \ldots, 4$, whose time-space evolution is due to free streaming as well as to elastic and reactive collisions. In the absence of external forces, the kinetic equations of the SRS model can be written in the form

$$
\frac{\partial f_{i}}{\partial t}+v \cdot \nabla_{x} f_{i}=J_{i}^{E}+J_{i}^{R}, \quad i=1, \ldots, 4,
$$

where $J_{i}^{E}$ and $J_{i}^{R}$ are the elastic and the reactive collision terms, given by

$$
\begin{aligned}
J_{i}^{E} & =\sum_{s=1}^{4}\left\{\sigma_{i s}^{2} \iint_{\mathbb{R}^{3} \times \mathbb{S}_{+}^{2}}\left[f_{i}\left(t, x, v^{\prime}\right) f_{s}\left(t, x, w^{\prime}\right)-f_{i}(t, x, v) f_{s}(t, x, w)\right]\langle\epsilon, v-w\rangle d \epsilon d w\right\} \\
& -\beta_{i j} \sigma_{i j}^{2} \iint_{\mathbb{R}^{3} \times \mathbb{S}_{+}^{2}}\left[f_{i}\left(t, x, v^{\prime}\right) f_{j}\left(t, x, w^{\prime}\right)-f_{i}(t, x, v) f_{j}(t, x, w)\right] \Theta\left(\langle\epsilon, v-w\rangle-\Gamma_{i j}\right)\langle\epsilon, v-w\rangle d \epsilon d w
\end{aligned}
$$

and

$$
\begin{aligned}
& J_{i}^{R}=\beta_{i j} \sigma_{i j}^{2} \iint_{\mathbb{R}^{3} \times \mathbb{S}_{+}^{2}}\left[\left(\frac{\mu_{i j}}{\mu_{k l}}\right)^{2} f_{k}\left(t, x, v_{i j}^{\odot}\right) f_{l}(\right.\left.\left., x, w_{i j}^{\odot}\right)-f_{i}(t, x, v) f_{j}(t, x, w)\right] \\
& \times \Theta\left(\langle\epsilon, v-w\rangle-\Gamma_{i j}\right)\langle\epsilon, v-w\rangle d \epsilon d w .
\end{aligned}
$$

In the above expressions of $J_{i}^{E}$ and $J_{i}^{R}$, the pairs of indices $(i, j)$ and $(k, l)$ are such that the corresponding quadruple $(i, j, k, l)$ is chosen according to (3). In the expression (5) of the elastic terms $J_{i}^{E}$, the second term singles out the fraction $\beta_{i j}$ of those pre-collisional states of $A_{i}$ and $A_{j}$ constituents that are energetic enough to produce a chemical reaction. This correction is specific to the SRS model where both elastic and reactive events are the results of hard-spheres particles with diameters $d_{i}, i=1, \ldots, 4$. Furthermore, $v$ and $w$ are pre-collisional velocities of 
particles from species $i$ and $s$, whereas $v^{\prime}$ and $w^{\prime}$ are the corresponding post-collisional velocities given by

$$
v^{\prime}=v-2 \frac{\mu_{i s}}{m_{i}} \epsilon\langle\epsilon, v-w\rangle, \quad w^{\prime}=w+2 \frac{\mu_{i s}}{m_{s}} \epsilon\langle\epsilon, v-w\rangle .
$$

Here, $\langle\cdot, \cdot\rangle$ is the inner product in $\mathbb{R}^{3}, \epsilon$ is a vector along the line passing through the centres of the spheres at the moment of impact, i.e., $\epsilon \in \mathbb{S}_{+}^{2}$, with $\mathbb{S}_{+}^{2}=\left\{\epsilon \in \mathbb{R}^{3}:|\epsilon|=1,\langle\epsilon, v-w\rangle \geq 0\right\}$, and $\mu_{i s}=m_{i} m_{s} /\left(m_{i}+m_{s}\right)$ is the reduced mass of the colliding pair. Moreover, $\Theta$ is the Heaviside step function, and $\Gamma_{i j}=\sqrt{2 \gamma_{i} / \mu_{i j}}$ represents a threshold velocity of the colliding pair.

Concerning expressions (6) of the reactive collision terms $J_{i}^{R}$, the pairs of post-reactive velocities associated to the endothermic forward reaction are $\left(v_{i j}^{\odot}, w_{i j}^{\odot}\right)=\left(v^{\ddagger}, w^{\ddagger}\right)$ for $i, j=1,2$, given by

$$
\begin{aligned}
v^{\ddagger} & =\frac{1}{M}\left[m_{1} v+m_{2} w+m_{4} \sqrt{\frac{\mu_{12}}{\mu_{34}}}\left\{(v-w)-\epsilon\langle\epsilon, v-w\rangle+\epsilon \alpha^{-}\right\}\right], \\
w^{\ddagger} & =\frac{1}{M}\left[m_{1} v+m_{2} w-m_{3} \sqrt{\frac{\mu_{12}}{\mu_{34}}}\left\{(v-w)-\epsilon\langle\epsilon, v-w\rangle+\epsilon \alpha^{-}\right\}\right],
\end{aligned}
$$

with $\alpha^{-}=\sqrt{(\langle\epsilon, v-w\rangle)^{2}-2 E_{a b s} / \mu_{12}}$. The post-reactive velocities associated to the exothermic backward reaction are $\left(v_{i j}^{\odot}, w_{i j}^{\odot}\right)=\left(v^{\dagger}, w^{\dagger}\right)$ for $i, j=3,4$, given by

$$
\begin{aligned}
v^{\dagger} & =\frac{1}{M}\left[m_{3} v+m_{4} w+m_{2} \sqrt{\frac{\mu_{34}}{\mu_{12}}}\left\{(v-w)-\epsilon\langle\epsilon, v-w\rangle+\epsilon \alpha^{+}\right\}\right], \\
w^{\dagger} & =\frac{1}{M}\left[m_{3} v+m_{4} w-m_{1} \sqrt{\frac{\mu_{34}}{\mu_{12}}}\left\{(v-w)-\epsilon\langle\epsilon, v-w\rangle+\epsilon \alpha^{+}\right\}\right],
\end{aligned}
$$

with $\alpha^{+}=\sqrt{(\langle\epsilon, v-w\rangle)^{2}+2 E_{a b s} / \mu_{34}}$.

Post- and pre-collisional velocities of the reactive pairs satisfy momentum conservation total energy conservation, that is

$$
\begin{array}{rlrl}
m_{1} v+m_{2} w & =m_{3} v^{\ddagger}+m_{4} w^{\ddagger}, & m_{3} v+m_{4} w & =m_{1} v^{\dagger}+m_{2} w^{\dagger}, \\
m_{1} v^{2}+m_{2} w^{2}=m_{3} v^{\ddagger 2}+m_{4} w^{\ddagger 2}+2 E_{a b s}, & m_{3} v^{2}+m_{4} w^{2}=m_{1} v^{\dagger 2}+m_{2} w^{\dagger 2}-2 E_{a b s},
\end{array}
$$

Finally, we point out that the SRS kinetic system considered in this work does not necessarily satisfy conservation of the angular momentum during reactive collisions, unless $\mu_{i j} / \mu_{k l}=1$. On the other hand, the modified SRS kinetic model considered in [37] has conservation of the angular momentum built into the collisional processes. The exponent 2 in the quotient $\mu_{i j} / \mu_{k l}$ of (6) for $J_{i}^{R}$ is specific to the SRS model considered here, while the corresponding exponent in expression for $J_{i}^{R}$ in the modified SRS model is equal to 3 .

Remark 2.1. (a) SRS kinetic system (4) reduces to the Boltzmann system for a non-reactive mixture with hard-spheres elastic collisions, when we turn off the chemical reaction. i.e., when coefficients $\beta_{i j}$ are taken equal to zero, for all $i, j=1, \ldots, 4$.

$$
\begin{aligned}
& \frac{\partial f_{i}}{\partial t}+v \cdot \nabla_{x} f_{i}=\sum_{s=1}^{4} \sigma_{i s}^{2} \iint_{\mathbb{R}^{3} \times \mathbb{S}_{+}^{2}}\left[f_{i}\left(t, x, v^{\prime}\right) f_{s}\left(t, x, w^{\prime}\right)-f_{i}(t, x, v) f_{s}(t, x, w)\right]\langle\epsilon, v-w\rangle d \epsilon d w, \\
& \text { for } \quad i=1, \ldots, 4 .
\end{aligned}
$$


In particular, the post collisional velocities $v^{\prime}$ and $w^{\prime}$ are given by expressions (7). Equations (12) will be used in this paper when studying the influence of the chemical reaction in the transport properties of the SRS kinetic system (4). For example, the viscosity coefficient of the reactive mixture described by Eqs. (4) will be normalized with respect to the one of the non-reactive mixture described by Eqs. (12).

(b) On the other hand, if the coefficients $\beta_{i j}$ are taken equal to one, for all $i, j=1, \ldots, 4$, the resulting SRS kinetic system (4) corresponds to the situation in which all collisions with sufficient amount of energy result in the chemical reactions. This limiting situation can be relevant when dealing with chemical reactions of ionized species [3, 25].

\subsection{Equilibrium}

For the chemically reactive mixture described by the SRS kinetic system $(4),(5),(6)$, the equilibrium solutions are distribution functions $f_{i}(t, x, v)$ satisfying

$$
J_{i}^{E}+J_{i}^{R}=0, \quad i=1, \ldots, 4 .
$$

This implies that the equilibrium distributions are Maxwellian centred at the mixture rest frame, given by

$$
f_{i}=n_{i}\left(\frac{m_{i}}{2 \pi k T}\right)^{3 / 2} \exp \left[-\frac{m_{i}(v-u)^{2}}{2 k T}\right], \quad i=1, \ldots, 4,
$$

with $n_{i}$ and temperature $T$ constrained to the condition $n_{1} n_{2}=\left(\mu_{12} / \mu_{34}\right)^{1 / 2} n_{3} n_{4} \exp \left(E_{a b s} /(k T)\right)$. Such condition can be interpreted as the requirement on $n_{i}$ and $T$ in order to assure the chemical equilibrium for the chemical reaction (1). It represents the mass action law of our SRS kinetic model, and has the form

$$
\frac{n_{1}^{\mathrm{eq}} n_{2}^{\mathrm{eq}}}{n_{3}^{\mathrm{eq}} n_{4}^{\mathrm{eq}}}=\left(\frac{\mu_{12}}{\mu_{34}}\right)^{1 / 2} \exp \left(\frac{E_{a b s}}{k T}\right),
$$

where $n_{i}^{\mathrm{eq}}$ represents the number density of each $i$-constituent referred to chemical equilibrium conditions.

From here on, we will use the notation $F_{i}^{M}$ to indicate equilibrium distributions defined as in (14) with number densities $n_{i}$ constrained by the mass action law. Furthermore, we will use the notation $f_{i}^{M}$ to indicate Maxwellian distributions defined as in (14) but with number densities not constrained by the mass action law.

Properties of the Maxwellian distributions. Using the conservation of kinetic energy during elastic collisions we obtain a useful relationship between the Maxwellians associated to pre and post velocities, $v, w$ and $v^{\prime}, w^{\prime}$, respectively. Omitting the dependence on $t$ and $x$, this relationship reads

$$
f_{i}^{M}\left(v^{\prime}\right) f_{s}^{M}\left(w^{\prime}\right)=f_{i}^{M}(v) f_{s}^{M}(w), \quad \text { for } \quad i, s=1, \ldots, 4 .
$$

Analogously, using the conservation of total energy during reactive encounters we also obtain

$$
\begin{aligned}
\left(\frac{\mu_{i j}}{\mu_{k l}}\right)^{2} f_{k}^{M}\left(v_{i j}^{\odot}\right) f_{l}^{M}\left(w_{i j}^{\odot}\right)-f_{i}^{M}(v) f_{j}^{M}(w) & =f_{i}^{M}(v) f_{j}^{M}(w)\left[\left(\frac{\mu_{i j}}{\mu_{k l}}\right)^{2} \frac{f_{k}^{M}\left(v_{i j}^{\odot}\right) f_{l}^{M}\left(w_{i j}^{\odot}\right)}{f_{i}^{M}(v) f_{j}^{M}(w)}-1\right] \\
& =f_{i}^{M}(v) f_{j}^{M}(w)\left[\left(\frac{\mu_{i j}}{\mu_{k l}}\right)^{1 / 2} \exp \left(\frac{E_{a b s}}{k T}\right) \frac{n_{k} n_{l}}{n_{i} n_{j}}-1\right] .
\end{aligned}
$$


Chemical affinity. The deviation of the reactive mixture from the chemical equilibrium can be measured by the chemical affinity $\mathcal{A}$ of the forward reaction, which is defined by (see Ref. [4])

$$
\mathcal{A}=k T \ln \left(\frac{n_{1} n_{2} n_{3}^{\mathrm{eq}} n_{4}^{\mathrm{eq}}}{n_{3} n_{4} n_{1}^{\mathrm{eq}} n_{2}^{\mathrm{eq}}}\right) .
$$

The forward affinity vanishes at equilibrium conditions and tends to $-\infty$ at the extreme conditions for which only the products of the forward reaction are present $\left(n_{1}=n_{2}=0\right)$. Using the mass action law (15) we obtain

$$
\exp \left(-\frac{\mathcal{A}}{k T}\right)=\left(\frac{\mu_{12}}{\mu_{34}}\right)^{1 / 2} \exp \left(\frac{E_{a b s}}{k T}\right) \frac{n_{3} n_{4}}{n_{1} n_{2}}
$$

which will be used in the sequel. The affinity $\mathcal{A}$ represents a thermodynamical force and plays an important role in the computation of the transport coefficients for a chemically reactive mixture, in the sense that the non-equilibrium deviations of the distribution functions $f_{i}$ are expressed in terms of $\mathcal{A}$ as well as in terms of other thermodynamical forces (see Eq. (39) of Subsection 3.2).

\subsection{Macroscopic field equations}

If we consider $n_{i}(i=1, \ldots, 4), u$, and $T$ as the hydrodynamic fields describing the reactive mixture, the evolution equations for such fields can be formally derived from the SRS system (446) in the form, see [37,

$$
\begin{aligned}
& \frac{\partial n_{i}}{\partial t}+\sum_{s=1}^{3} \frac{\partial}{\partial x_{s}}\left(n_{i} \bar{u}_{i}^{(s)}+n_{i} u^{(s)}\right)=\tau_{i}, \quad \text { with } \quad \tau_{i}=\int_{\mathbb{R}^{3}} J_{i}^{R} d v, \quad \text { for } \quad i=1, \ldots, 4 \\
& \frac{\partial}{\partial t}\left(\varrho u^{(r)}\right)+\sum_{s=1}^{3} \frac{\partial}{\partial x_{s}}\left[p^{(r s)}+\varrho u^{(r)} u^{(s)}\right]=0, \quad \text { for } \quad r=1,2,3, \\
& \frac{\partial}{\partial t}\left(\frac{3 n k T}{2}+\sum_{i=1}^{4} n_{i} E_{i}+\frac{\varrho u^{2}}{2}\right)+\sum_{r=1}^{3} \frac{\partial}{\partial x_{r}}\left[q^{(r)}+\sum_{s=1}^{3} p^{(r s)} u^{(s)}+\left(\frac{3 n k T}{2}+\sum_{i=1}^{4} n_{i} E_{i}+\frac{\varrho u^{2}}{2}\right) u^{(r)}\right]=0
\end{aligned}
$$

Here, the symbol $\bar{u}_{i}$ denotes the diffusion velocity of the ith component of the reactive mixture, and $n, \varrho, u, p^{(r s)}, p$, and $q$ represent, respectively, the number density, mass density, mean velocity, pressure tensor components, pressure and heat flux of the mixture. The upper indices $r$ and $s$ indicate spatial directions in a given orthogonal reference system. In our analysis developed in the next sections, the pressure tensor will be of special interest. It can be defined in terms of its spatial components $p^{(r s)}$ by

$$
p^{(r s)}(t, x)=\sum_{i=1}^{4} \int_{\mathbb{R}^{3}}\left(v_{r}-u_{r}(t, x)\right)\left(v_{s}-u_{s}(t, x)\right) f_{i}(t, x, v) d v, \quad r, s=1,2,3 .
$$

We do not give here the explicit expressions for the other quantities, the reader is referred to paper [37].

Equation (20) is the chemical rate equation of the SRS model, and specifies the balance of the number density of each i-th component of the mixture. The production term $\tau_{i}$ on its right-hand 
side represents the reaction rate for the i-th component and is given by

$$
\begin{array}{r}
\tau_{i}(t, x)=-\nu_{i} \beta_{12} \sigma_{12}^{2} \iiint_{\mathbb{R}^{3} \times \mathbb{R}^{3} \times \mathbb{S}_{+}^{2}}\left[\left(\frac{\mu_{12}}{\mu_{34}}\right)^{2} f_{3}\left(t, x, v_{12}^{\odot}\right) f_{4}\left(t, x, w_{12}^{\odot}\right)-f_{1}(t, x, v) f_{2}(t, x, w)\right] \\
\times \Theta\left(\langle\epsilon, v-w\rangle-\Gamma_{12}\right)\langle\epsilon, v-w\rangle d \epsilon d w d v, \quad i=1, \ldots, 4,
\end{array}
$$

where the coefficients $\nu_{i}$ are the stoichiometric coefficients associated to the chemical reaction (1), with $\nu_{1}=\nu_{2}=-1, \nu_{3}=\nu_{4}=1$. Equations (21) and 222) are the conservation laws for the momentum components and total energy of the mixture, respectively.

As usual, Eqs. (20), 21) and (22) do not constitute a closed system for the macroscopic evolution of the reactive mixture, because it involves the unknown fields of diffusion velocities $\bar{u}_{i}$, pressure tensor $p^{(r s)}$, heat flux $q$, as well as the chemical reaction rate $\tau_{i}$. To close such system, one can use the Chapman-Enskog method to determine an approximate solution to the SRS system (4), (5) and (6) and then compute the unknown terms in the macroscopic equations. This will be the object of the next section.

\section{The transport algorithm}

In this section, we determine an approximate solution to the SRS system (4), (5) and (6), using the Chapman-Enskog method [3, 4, 38, 39]. Being associated with a chemically reactive mixture, such approximate solution should contain the non-equilibrium effects induced by the chemical reaction.

We consider a chemical regime for which the reaction process is close to the chemical equilibrium and the chemical characteristic time is of the same order of magnitude as the characteristic time of the non-reactive (elastic) processes. This regime corresponds to a fast chemical reaction, and has been formally introduced in paper [9] and then considered in papers [18, 23, 27, 28] for reactive mixtures of two or four constituents.

\subsection{The Chapman-Enskog procedure}

Following papers [9, 16, 18, 27], we start with the SRS kinetic system (4) written in dimensionless form,

$$
\frac{\partial f_{i}}{\partial t}+v \cdot \nabla_{x} f_{i}=\frac{1}{\epsilon}\left(J_{i}^{E}+J_{i}^{R}\right), \quad i=1, \ldots, 4,
$$

where $\epsilon$ is a formal expansion parameter and consider a first-order expansion of the distribution functions $f_{i}$ of the form

$$
f_{i}=f_{i}^{(0)}\left(1+\epsilon \phi_{i}\right), \quad i=1, \ldots, 4,
$$

where $\phi_{i}=\phi_{i}(t, x, v)$ represents a small perturbation function. Introducing expansions (26) into Eqs. 25 and equating the terms of the same order in $\epsilon$, we obtain the integral equations that should be satisfied by both approximations $f_{i}^{(0)}$ and perturbations $\phi_{i}$. 
Furthermore, the Chapman-Enskog method requires that $f_{i}$ and $f_{i}^{(0)}$ yield the same local macroscopic quantities (moments of order $0,1,2$ ), and thus we have that

$$
\begin{aligned}
\sum_{i=1}^{4} \int_{\mathbb{R}^{3}} f_{i}(t, x, v) d v & =\sum_{i=1}^{4} \int_{\mathbb{R}^{3}} f_{i}^{(0)}(t, x, v) d v \\
\sum_{i=1}^{4} \int_{\mathbb{R}^{3}} m_{i} v f_{i}(t, x, v) d v & =\sum_{i=1}^{4} \int_{\mathbb{R}^{3}} m_{i} v f_{i}^{(0)}(t, x, v) d v \\
\sum_{i=1}^{4} \int_{\mathbb{R}^{3}}\left(m_{i} \frac{v^{2}}{2}+E_{i}\right) f_{i}(t, x, v) d v & =\sum_{i=1}^{4} \int_{\mathbb{R}^{3}}\left(m_{i} \frac{v^{2}}{2}+E_{i}\right) f_{i}^{(0)}(t, x, v) d v .
\end{aligned}
$$

Conditions (27) imply that the $\phi_{i}$ obey the following constraints that will be essential in the approximation procedure,

$$
\sum_{i=1}^{4} \int_{\mathbb{R}^{3}} f_{i}^{(0)} \phi_{i} d v=0, \quad \sum_{i=1}^{4} \int_{\mathbb{R}^{3}} m_{i} v f_{i}^{(0)} \phi_{i} d v=0, \quad \sum_{i=1}^{4} \int_{\mathbb{R}^{3}}\left(m_{i} \frac{v^{2}}{2}+E_{i}\right) f_{i}^{(0)} \phi_{i} d v=0 .
$$

Zero-order approximation. Equating the terms of the order $\mathcal{O}(1 / \epsilon)$ in Eqs. (25), we obtain the following equations for $f_{i}^{(0)}$,

$$
J_{i}^{E}\left(\left\{f_{i}^{(0)}\right\}\right)+J_{i}^{R}\left(\left\{f_{i}^{(0)}\right\}\right)=0, \quad i=1, \ldots, 4,
$$

where the notations $J_{i}^{E}\left(\left\{f_{i}^{(0)}\right\}\right)$ and $J_{i}^{R}\left(\left\{f_{i}^{(0)}\right\}\right)$ signify the fact that, for each $i=1, \ldots, 4$, the collision operators depend on $f_{1}^{(0)}, f_{2}^{(0)}, f_{3}^{(0)}, f_{4}^{(0)}$. Equations 29 identify the zero-order approximations $f_{i}^{(0)}$ as the Maxwellians (14) that assure the chemical equilibrium of the reactive mixture. Therefore, the number densities of the constituents at this order of approximation, $n_{i}^{(0)}$, $i=1, \ldots, 4$, are constrained to the mass action law (15), and we have

$$
\frac{n_{1}^{(0)} n_{2}^{(0)}}{n_{3}^{(0)} n_{4}^{(0)}}=\left(\frac{\mu_{12}}{\mu_{34}}\right)^{1 / 2} \exp \left(\frac{E_{a b s}}{k T}\right)
$$

The macroscopic equations at the Euler level express conservation of number densities of the constituents and of momentum and total energy of the mixture.

Time derivative of the zero-order approximation. From expressions (14) of the Maxwellian number densities, combined with the mass action law (30) and Euler equations, we can evaluate the time derivative of $f_{i}^{(0)}$, which will be useful in the sequel. We obtain

$$
\begin{aligned}
\frac{\partial f_{i}^{(0)}}{\partial t}+v \cdot \nabla_{x} f_{i}^{(0)} & =f_{i}^{(0)}\left\{\frac{m_{i}}{k T} \sum_{r, s}^{3}\left(v_{r}-u_{r}\right)\left(v_{s}-u_{s}\right)\left(\frac{\partial u_{s}}{\partial x_{r}}-\frac{1}{3}\left(\sum_{\ell}^{3} \frac{\partial u_{\ell}}{\partial x_{\ell}}\right) \delta_{r s}\right)\right. \\
+ & \sum_{r=1}^{3} \frac{1}{T}\left(v_{r}-u_{r}\right)\left(\frac{m_{i}}{2 k T}(v-u)^{2}-\frac{5}{2}\right) \frac{\partial T}{\partial x_{r}}+\frac{n}{n_{i}} \sum_{r=1}^{3}\left(v_{r}-u_{r}\right) d_{r}^{i} \\
+ & \left.\frac{\nu_{i}}{n_{i}} \tau_{f}^{(0)}+\frac{1}{p}\left(1-\frac{m_{i}}{3 k T}(v-u)^{2}\right) \sum_{i=1}^{4} \nu_{i} E_{i} \tau_{f}^{(0)}\right\},
\end{aligned}
$$


where $d_{r}^{i}$ represents spatial $r$-components of generalized diffusion forces of the $i$-constituent, given by

$$
d_{r}^{i}=\frac{1}{p} \frac{\partial p_{i}}{\partial x_{r}}-\frac{\rho_{i}}{\rho p} \frac{\partial p}{\partial x_{r}}, \quad i=1, \ldots, 4, \quad r=1,2,3,
$$

obeying the constraint

$$
\sum_{i=1}^{4} d_{r}^{i}=0, \quad r=1,2,3,
$$

and $\tau_{f}^{(0)}$ represents the forward reaction rate at the zero-order level, given by

$$
\tau_{f}^{(0)}=k_{f}^{(0)} n_{1}^{(0)} n_{2}^{(0)}
$$

with $k_{f}^{(0)}$ being the rate constant at the zero-order level,

$$
k_{f}^{(0)}=\beta_{12} \sigma_{12}^{2} \sqrt{\frac{8 \pi k T}{\mu_{12}}} \exp \left(-\frac{\gamma_{1}}{k T}\right),
$$

and $\gamma_{1}$ the activation energy of the forward reaction, as introduced in Section 2 .

First-order approximation. Equating the terms of the order $\mathcal{O}(1)$ in Eqs. (25), we obtain the following linear integral equations for the perturbation terms $\phi_{i}$,

$$
\begin{aligned}
& f_{i}^{(0)}\left\{\frac{m_{i}}{k T} \sum_{r, s}^{3}\left(v_{r}-u_{r}\right)\left(v_{s}-u_{s}\right)\left(\frac{\partial u_{s}}{\partial x_{r}}-\frac{1}{3}\left(\sum_{\ell}^{3} \frac{\partial u_{\ell}}{\partial x_{\ell}}\right) \delta_{r s}\right)\right. \\
& +\sum_{r=1}^{3} \frac{1}{T}\left(v_{r}-u_{r}\right)\left(\frac{m_{i}}{2 k T}(v-u)^{2}-\frac{5}{2}\right) \frac{\partial T}{\partial x_{r}}+\frac{n}{n_{i}} \sum_{r=1}^{3}\left(v_{r}-u_{r}\right) d_{r}^{i} \\
& +\frac{\nu_{i}}{n_{i}} \tau_{f}^{(0)} \frac{\mathcal{A}}{k T}-\frac{2 \nu_{i}}{3 n} \tau_{f}^{(0)} \frac{E_{a b s}}{k T}\left(\frac{3}{2}-\frac{m_{i}}{2 k T}(v-u)^{2}\right) \frac{\mathcal{A}}{k T} \\
& \left.+\beta_{i j} \sigma_{i j}^{2}\left(\iint_{\mathbb{R}^{3} \times \mathbb{S}_{+}^{2}} f_{j}^{(0)}(w) \Theta\left(\langle\epsilon, v-w\rangle-\Gamma_{i j}\right)\langle\epsilon, v-w\rangle d \epsilon d w\right) \frac{\mathcal{A}}{k T}\right\} \\
& =\mathscr{L}_{i}^{E}\left(\left\{\phi_{i}\right\}\right)+\mathscr{L}_{i}^{R}\left(\left\{\phi_{i}\right\}\right), \quad \text { for } \quad i=1, \ldots, 4,
\end{aligned}
$$

where $\mathscr{L}_{i}^{E}$ and $\mathscr{L}_{i}^{R}$ are the linearized elastic and reactive collisional operators and the notations $\mathscr{L}_{i}^{E}\left(\left\{\phi_{i}\right\}\right)$ and $\mathscr{L}_{i}^{R}\left(\left\{\phi_{i}\right\}\right)$, as before, signify the fact that, for each $i=1, \ldots, 4$, the linearized operators depend on $\phi_{1}, \phi_{2}, \phi_{3}, \phi_{4}$. Operators $\mathscr{L}_{i}^{E}$ and $\mathscr{L}_{i}^{R}$ are defined by 


$$
\begin{aligned}
\mathscr{L}_{i}^{E}(\phi)= & \sum_{s=1}^{4} \sigma_{i s}^{2} \iint_{\mathbb{R}^{3} \times \mathbb{S}_{+}^{2}}\left[f_{i}^{(0)}(v) f_{s}^{(0)}(w)\left(\phi_{i}\left(v^{\prime}\right)+\phi_{s}\left(w^{\prime}\right)-\phi_{i}(v)-\phi_{s}(w)\right)\right]\langle\epsilon, v-w\rangle d \epsilon d w \\
& -\beta_{i j} \sigma_{i j}^{2} \iint_{\mathbb{R}^{3} \times \mathbb{S}_{+}^{2}}\left[f_{i}^{(0)}(v) f_{j}^{(0)}(w)\left(\phi_{i}\left(v^{\prime}\right)+\phi_{j}\left(w^{\prime}\right)-\phi_{i}(v)-\phi_{j}(w)\right)\right] \\
& \times \Theta\left(\langle\epsilon, v-w\rangle-\Gamma_{i j}\right)\langle\epsilon, v-w\rangle d \epsilon d w \\
\mathscr{L}_{i}^{R}(\phi)= & \beta_{i j} \sigma_{i j}^{2} \iint_{\mathbb{R}^{3} \times \mathbb{S}_{+}^{2}}\left[f_{i}^{(0)}(v) f_{j}^{(0)}(w)\left(\phi_{k}\left(v_{i j}^{\odot}\right)+\phi_{l}\left(w_{i j}^{\odot}\right)-\phi_{i}(v)-\phi_{j}(w)\right)\right] \\
& \times \Theta\left(\langle\epsilon, v-w\rangle-\Gamma_{i j}\right)\langle\epsilon, v-w\rangle d \epsilon d w .
\end{aligned}
$$

\subsection{The perturbations $\phi_{i}$}

Equations (36) constitute a non-homogeneous linear system of coupled integral equations for the perturbations $\phi_{i}, i=1,2,3,4$. They have to be solved together with the constraints (28) on $\phi_{i}$ in order to determine $\phi_{i}$ uniquely.

From the analysis of the left-hand side of Eq. (36), a good trial form for the perturbation $\phi_{i}$ is a linear combination of the thermodynamic forces appearing on the left-hand side, namely the gradient of temperature, the velocity gradient deviator, the generalized diffusion forces and the affinity. These are independent forces (causes) that will produce the thermodynamic fluxes (effects). Thus, we assume a solution for $\phi_{i}$ in the form

$$
\begin{aligned}
\phi_{i}=-A_{i} \sum_{r=1}^{3}\left(v_{r}-u_{r}\right) \frac{\partial T}{\partial x_{r}}-B_{i} \sum_{r, s}^{3}\left(v_{r}-u_{r}\right)\left(v_{s}-u_{s}\right)\left(\frac{\partial u_{s}}{\partial x_{r}}-\frac{1}{3}\left(\sum_{\ell}^{3} \frac{\partial u_{\ell}}{\partial x_{\ell}}\right) \delta_{r s}\right) & \\
& -\sum_{j=1}^{4} F_{i j} \sum_{r=1}^{3}\left(v_{r}-u_{r}\right) d_{r}^{j}-G_{i} \frac{\mathcal{A}}{k T}, \quad i=1, \ldots, 4,
\end{aligned}
$$

where the coefficients $A_{i}, B_{i}, F_{i j}$ and $G_{i}$ are scalar quantities depending on the number densities $n_{i}$, scalar velocity $|v-u|^{2}$, and mixture temperature $T$. Therefore, the problem of determining the perturbation functions $\phi_{i}$ becomes the one of determining such coefficients. The constraints (28) on $\phi_{i}$ and (33) on the diffusion forces lead to further conditions that have to be satisfied by the unknown coefficients.

As usual in the Chapman-Enskog method [38], the next step consists in inserting expansion (39) into the integral equations (36) and then using the fact that the thermodynamic forces appearing in the expansion are linearly independent. Therefore system (36) decouples into four subsystems, and for our convenience, we introduce the following notation for the components of 
$\phi_{i}$,

$$
\begin{aligned}
\phi_{i}^{(A)} & =A_{i} \sum_{r=1}^{3}\left(v_{r}-u_{r}\right) \frac{\partial T}{\partial x_{r}}, \quad \phi_{i}^{(B)}=B_{i} \sum_{r, s}^{3}\left(v_{r}-u_{r}\right)\left(v_{s}-u_{s}\right)\left(\frac{\partial u_{s}}{\partial x_{r}}-\frac{1}{3}\left(\sum_{\ell}^{3} \frac{\partial u_{\ell}}{\partial x_{\ell}}\right) \delta_{r s}\right), \\
\phi_{i}^{(F)} & =\sum_{j=1}^{4} F_{i j} \sum_{r=1}^{3}\left(v_{r}-u_{r}\right) d_{r}^{j}, \quad \phi_{i}^{(G)}=G_{i} \frac{\mathcal{A}}{k T}, \quad i=1, \ldots, 4 .
\end{aligned}
$$

One subsystem involves the component $\phi_{i}^{(A)}$ and determines the coefficients $A_{i}$, another one involves the component $\phi_{i}^{(B)}$ and determines the coefficients $B_{i}$, and analogously for the components $\phi_{i}^{(F)}, \phi_{i}^{(G)}$ and coefficients $F_{i j}, G_{i}$. The resulting subsystems are solved separately assuming appropriate expansions for the coefficients $A_{i}, B_{i}, F_{i j}$ and $G_{i}$. A standard assumption in the Chapman-Enskog method is to consider that such coefficients are expanded in terms of specific Sonine polynomials of the dimensionless quantity,

$$
C_{i}^{2}=m_{i} \frac{(v-u)^{2}}{2 k T}
$$

Exploiting the orthogonality of the polynomials, the equations of each subsystem are then multiplied by specific Sonine polynomials and then integrated over the velocity space. For a detailed explanation about the solution procedure see, for example, Refs. [3, 4, 38, 39].

\section{Transport coefficients}

In the present work, we are interested in the coefficients of the reaction rate and shear viscosity. We will apply the solution procedure described in the previous section omitting the computational details.

Reaction rate coefficients. The forward and backward reaction rate coefficients are obtained solving the subsystem for the coefficients $G_{i}$ associated to the perturbation component $\phi_{i}^{(G)}$, and then using the corresponding deviation $f_{i}^{(0)} \phi_{i}^{(G)}$ to determine the first-order correction to the reaction rate $\tau_{i}^{(0)}$. The subsystem for $G_{i}$ reads

$$
\begin{aligned}
f_{i}^{(0)} & \left\{\frac{\nu_{i}}{n_{i}} \tau_{f}^{(0)}-\frac{2 \nu_{i}}{3 n} \tau_{f}^{(0)} \frac{E_{a b s}}{k T}\left(\frac{3}{2}-\frac{m_{i}}{2 k T}(v-u)^{2}\right)\right. \\
& \left.+\beta_{i j} \sigma_{i j}^{2}\left(\iint_{\mathbb{R}^{3} \times \mathbb{S}_{+}^{2}} f_{j}^{(0)}(w) \Theta\left(\langle\epsilon, v-w\rangle-\Gamma_{i j}\right)\langle\epsilon, v-w\rangle d \epsilon d w\right)\right\} \frac{\mathcal{A}}{k T} \\
& =\mathscr{L}_{i}^{E}\left(\left\{\phi_{i}^{(G)}\right\}\right)+\mathscr{L}_{i}^{R}\left(\left\{\phi_{i}^{(G)}\right\}\right), \quad \text { for } \quad i=1, \ldots, 4 .
\end{aligned}
$$

As usual, we assume that each coefficient $G_{i}$ is expanded in terms of Sonine polynomials of index $1 / 2$ and the first two terms of the expansion are adequate for the first-order approximation of the reaction rate coefficient. Thus we start with

$$
G_{i}=g_{i}^{(0)} S_{1 / 2}^{(0)}\left(C_{i}^{2}\right)+g_{i}^{(1)} S_{1 / 2}^{(1)}\left(C_{i}^{2}\right), \quad \text { that is } G_{i}=g_{i}^{(0)}+g_{i}^{(1)}\left(\frac{3}{2}-\frac{m_{i}}{2 k T}(v-u)^{2}\right), \quad i=1,2,3,4,
$$


where $g_{i}^{(0)}$ and $g_{i}^{(1)}$ are scalar constants to be determined. From constraints (28), we obtain

$$
g_{i}^{(0)}=0, \text { for } i=1,2,3,4, \quad \text { and } \quad \sum_{i=1}^{4} n_{i} g_{i}^{(1)}=0 .
$$

The scalars $g_{i}^{(1)}$ are determined by multiplying Eq. (41) by the polynomial $S_{1 / 2}^{(1)}\left(C_{i}^{2}\right)$, integrating first over $v \in \mathbb{R}^{3}$ and then over $\mathbb{S}_{+}^{2}$ by using spherical coordinates and finally computing the sixfold integral over $\mathbb{R}^{3} \times \mathbb{R}^{3}$ by transforming to the center of mass reference frame. The resulting equations for the scalars $g_{i}^{(1)}$ constitute an algebraic system, omitted here for brevity. Such an algebraic system can be solved using any computer algebra system and we have used the Maple software, version 2015. The solution of the system determines the perturbation component $\phi_{i}^{(G)}$ through Eqs. 40 and 42 .

The first-order correction to the forward and backward reaction rates can be computed by inserting the distribution function corrected by its affinity component, $f_{i}^{(0)}\left(1+\phi_{i}^{(G)}\right)$, into expression (24) of the reaction rate. Performing the integration over the velocity $v$ and neglecting the non-linear terms in the perturbations, we obtain the reaction rate law of our model

$$
\tau_{i}^{(1)}=\nu_{i}\left(k_{f}^{(1)} n_{1}^{(0)} n_{2}^{(0)}-k_{b}^{(1)} n_{3}^{(0)} n_{4}^{(0)}\right) \frac{\mathcal{A}}{k T}, \quad i=1, \ldots, 4,
$$

with the forward and backward reaction rate constants, given by

$$
\begin{aligned}
& k_{f}^{(1)}=-\frac{\beta_{12} \sigma_{12}^{2}}{n_{1}^{(0)} n_{2}^{(0)}} \iiint_{\mathbb{R}^{3} \times \mathbb{R}^{3} \times \mathbb{S}_{+}^{2}} f_{1}^{(0)} f_{2}^{(0)}\left(G_{1}+G_{2}\right) \Theta\left(\langle\epsilon, v-w\rangle-\Gamma_{i j}\right)\langle\epsilon, v-w\rangle d \epsilon d w d v, \\
& k_{b}^{(1)}=-\frac{\beta_{34} \sigma_{34}^{2}}{n_{3}^{(0)} n_{4}^{(0)}} \iiint_{\mathbb{R}^{3} \times \mathbb{R}^{3} \times \mathbb{S}_{+}^{2}}\left(\frac{\mu_{12}}{\mu_{34}}\right)^{2} f_{3}^{(0)} f_{4}^{(0)}\left(G_{3}+G_{4}\right) \Theta\left(\langle\epsilon, v-w\rangle-\Gamma_{i j}\right)\langle\epsilon, v-w\rangle d \epsilon d w d v .
\end{aligned}
$$

Shear viscosity coefficient. The shear viscosity coefficient is obtained by solving the following subsystem for the coefficients $B_{i}$ that are associated with the perturbation component $\phi_{i}^{(B)}$. Next, we use the corresponding deviation $f_{i}^{(0)} \phi_{i}^{(B)}$ to determine the first-order correction of the pressure tensor $p^{(r s)}$. The subsystem for $B_{i}$ reads

$$
\begin{aligned}
f_{i}^{(0)}\left\{\frac { m _ { i } } { k T } \sum _ { r , s } ^ { 3 } \left(v_{r}\right.\right. & \left.\left.-u_{r}\right)\left(v_{s}-u_{s}\right)\left(\frac{\partial u_{s}}{\partial x_{r}}-\frac{1}{3}\left(\sum_{\ell}^{3} \frac{\partial u_{\ell}}{\partial x_{\ell}}\right) \delta_{r s}\right)\right\} \\
= & \mathscr{L}_{i}^{E}\left(\left\{\phi_{i}^{(B)}\right\}\right)+\mathscr{L}_{i}^{R}\left(\left\{\phi_{i}^{(B)}\right\}\right), \quad \text { for } \quad i=1, \ldots, 4 .
\end{aligned}
$$

Now, we assume that each coefficient $B_{i}$ is expanded in terms of Sonine polynomials of index $5 / 2$, and thus

$$
B_{i}=b_{i}^{(0)} S_{5 / 2}^{(0)}\left(C_{i}^{2}\right), \quad \text { that is } B_{i}=b_{i}^{(0)}, \quad i=1,2,3,4 .
$$

Next, we multiply Eq. (46) by $S_{5 / 2}^{(1)}\left(C_{i}^{2}\right) \sum_{r, s}^{3}\left(v_{r}-u_{r}\right)\left(v_{s}-u_{s}\right)$ and integrate over $v \in \mathbb{R}^{3}$. The resulting algebraic equations determine the scalars $b_{i}^{(0)}$ and the perturbation component $\phi_{i}^{(B)}$ is completely known from (40) and (47). 
The first-order correction of the pressure tensor can be determined inserting the distribution function $f_{i}^{(0)}\left(1+\phi_{i}^{(B)}\right)$, with its viscous correction component, in expression $(23)$ of $p^{(r s)}$. Performing the integration in $v$ and neglecting the non-linear terms, we obtain the Navier-Stokes law for our model with the shear viscosity coefficient given by

$$
\mu=\sum_{i=1}^{4} n_{i} b_{i}^{(0)} k T .
$$

\section{$5 \quad$ Numerical computations}

In this section we present our results for a chemically reacting mixture described by the SRS model introduced in Section 2. We apply the transport algorithm presented in Section 3 and evaluate the coefficients of the reaction rate and shear viscosity as described in Section 4. Our aim is to analyse the influence of the chemical reaction in the behaviour of the forward and global reaction rates as well as in the behaviour of the shear viscosity coefficient. We also investigate the role of the elastic correction term included in the SRS model in order to appreciate how it can affects the transport coefficients.

\subsection{About the input data}

We consider different reactive mixtures, $\mathcal{M}_{1}, \ldots, \mathcal{M}_{12}$, specified by the molecular masses $m_{i}$ and equilibrium concentrations $x_{i}$ of the constituents, where $x_{i}=n_{i} / n$, for $i=1, \ldots, 4$. In Table 5.1 we present the model parameters characterizing these mixtures. In the last column of Table 5.1. we indicate the values of the reaction heat in units of $k T$. When $E_{a b s}<0$, the forward reaction is exothermic, and when $E_{a b s}>0$, it is endothermic.

\begin{tabular}{|c|cccc|cc|cccc|c|}
\hline Mixtures & $m_{1}$ & $m_{2}$ & $m_{3}$ & $m_{4}$ & $\mu_{12}$ & $\mu_{34}$ & $x_{1}$ & $x_{2}$ & $x_{3}$ & $x_{4}$ & $E_{\text {abs }} /(k T)$ \\
\hline \hline $\mathcal{M}_{1}$ & 2.2 & 1.8 & 2.8 & 1.2 & 0.99 & 0.84 & 0.10 & 0.10 & 0.40 & 0.40 & -2.855 \\
$\mathcal{M}_{2}$ & 2.2 & 1.8 & 2.8 & 1.2 & 0.99 & 0.84 & 0.40 & 0.40 & 0.10 & 0.10 & 2.690 \\
\hline $\mathcal{M}_{3}$ & 2.2 & 1.8 & 3 & 1 & 0.99 & 0.75 & 0.10 & 0.10 & 0.40 & 0.40 & -2.911 \\
$\mathcal{M}_{4}$ & 2.2 & 1.8 & 3 & 1 & 0.99 & 0.75 & 0.40 & 0.40 & 0.10 & 0.10 & 2.634 \\
\hline $\mathcal{M}_{5}$ & 2.2 & 1.8 & 3.2 & 0.8 & 0.99 & 0.64 & 0.10 & 0.10 & 0.40 & 0.40 & -2.991 \\
$\mathcal{M}_{6}$ & 2.2 & 1.8 & 3.2 & 0.8 & 0.99 & 0.64 & 0.40 & 0.40 & 0.10 & 0.10 & 2.555 \\
\hline $\mathcal{M}_{7}$ & 2.2 & 1.8 & 3.4 & 0.6 & 0.99 & 0.51 & 0.10 & 0.10 & 0.40 & 0.40 & -3.104 \\
$\mathcal{M}_{8}$ & 2.2 & 1.8 & 3.4 & 0.6 & 0.99 & 0.51 & 0.40 & 0.40 & 0.10 & 0.10 & 2.441 \\
\hline $\mathcal{M}_{9}$ & 2.2 & 1.8 & 3.6 & 0.4 & 0.99 & 0.36 & 0.10 & 0.10 & 0.40 & 0.40 & -3.278 \\
$\mathcal{M}_{10}$ & 2.2 & 1.8 & 3.6 & 0.4 & 0.99 & 0.36 & 0.40 & 0.40 & 0.10 & 0.10 & 2.267 \\
\hline $\mathcal{M}_{11}$ & 2.2 & 1.8 & 3.8 & 0.2 & 0.99 & 0.19 & 0.10 & 0.10 & 0.40 & 0.40 & -3.598 \\
$\mathcal{M}_{12}$ & 2.2 & 1.8 & 3.8 & 0.2 & 0.99 & 0.19 & 0.40 & 0.40 & 0.10 & 0.10 & 1.947 \\
\hline
\end{tabular}

Table 1: Different reactive mixtures considered in our computations.

The choice of the parameters and the equilibrium compositions of the reactive systems is presented below, together with the motivation for our choices. 
- We choose $x_{2}=x_{1}$ and $x_{4}=x_{3}$ for all computations, in agreement to other previous papers, in particular with [13] and [27]. Our choice is motivated by two factors. First, among the four concentrations $x_{i}$ only two are actually independent, because $\sum_{i=1}^{4} x_{i}=1$ and the mass action law furnishes other constraint on the values of $x_{i}$. Second, the ratios $x_{1} / x_{2}$ and $x_{3} / x_{4}$ of reactants and products do not have a great influence on the nature of the chemical reaction, as explained in paper [13].

- We fix $d_{i}=1, i=1, \ldots, 4$, for all computations, since the molecular diameters are not relevant in the chemical reactions in the dilute gas regime.

- We choose the molecular masses varying in such way that all constituents have different and possibly very disparate masses, in the sense that the reduced mass of the products can be $84.8 \%, 75.8 \%, 64.6 \%, 51.5 \%, 36.4 \%, 19.2 \%$ of that of the reactants. Moreover, due to the mass conservation, the masses should satisfy the condition $m_{1}+m_{2}=m_{3}+m_{4}$.

- The reaction heat $E_{a b s}$ is an important parameter in the dynamics of the chemical reaction. Its sign defines the nature of the reaction. Indeed, the forward reaction is exothermic if $E_{a b s}>0$ or endothermic if $E_{a b s}<0$. In our simulations this parameter is computed at equilibrium conditions from the mass action law (15), and thus from the molecular masses and equilibrium concentrations, as in

$$
\frac{E_{a b s}}{k T}=\ln \left(\sqrt{\frac{m_{3} m_{4}}{m_{1} m_{2}}} \frac{x_{1} x_{2}}{x_{3} x_{4}}\right) .
$$

Therefore the reaction heat $E_{a b s}$ is fixed when the molecular masses $m_{i}$ and concentrations $x_{i}$ are specified.

- The activation energies $\gamma_{1}$ and $\gamma_{3}$ are other important parameters in the dynamics of the chemical reaction, because they represent the energy barriers that should be exceeded in order for the forward and backward chemical reactions to occur. They are associated with the reaction heat $E_{a b s}$ by the relation $\gamma_{3}=\gamma_{1}-E_{a b s}$. This means that only one of the activation energies is an independent parameter; we choose $\gamma_{1}$ to be the independent one. In our simulations, we vary the forward activation energy in a wide range and investigate the behaviour of the transport coefficients in dependence on $\gamma_{1}$.

- Finally, the coefficients $\beta_{i j}, i, j=1, \ldots, 4$, which play the role of steric factors, are other important parameters in the description of the reactive mixture. We choose $\beta_{i j}=\beta_{k l}=\beta$ in our simulations because of the choice we made about the molecular diameters $d_{i}$. Moreover, we are considering a chemical regime for which the characteristic times of both elastic and reactive events are of the same order, and this implies that both elastic and reactive collisions occur with comparable frequencies, and therefore we assume $\beta=0.5,0.75,1$. More precisely, we consider $\beta=1$ when investigating the effects of the correction term on the transport coefficients, whereas we take $\beta=0.5,0.75,1$ when investigating the effect of such parameter on variability of the transport coefficients. These choices are essentially justified by the theoretical interest of our analysis for an abstract mixture.

\subsection{Results and discussion}

We report the results of our computations in the following subsections, starting with the results for non-equilibrium forward reaction rate, and followed by the results for the shear viscosity 
coefficient. A qualitative analysis is developed in order to discuss the results and how they are influenced by varying the model parameters.

We perform several computations for different choices of the molecular masses $m_{i}$ and equilibrium concentrations $x_{i}$, and also for different choices of the coefficient $\beta$ specifying various configurations of the SRS model in terms of the proportion of binary encounters that produce chemical reaction. This parametric study was implemented for all mixtures described in Table 5.1. but we choose only the more representative cases to illustrate our results in the figures presented below.

Our aim is to analyse two problems. First, we want to understand how the parameters $m_{i}, x_{i}$, and also the corresponding exothermicity or endothermicity character of the chemical reaction, influence the behaviour of the transport coefficients. Second, we want to investigate the impact of the correction term incorporated in our SRS model on the transport coefficients.

Comparisons with the results obtained with other models available in literature are also included, however comparisons with real physical systems and experimental works are not included, in fact this is not the objective of this paper.

\section{I) Results about the forward reaction rate.}

In order to estimate the non-equilibrium contributions to the forward reaction rate, we estimate the non-dimensional forward reaction rate using the non-dimensional rate constant

$$
k_{f}^{*}=k_{f}^{(1)} / k_{f}^{(0)},
$$

with $k_{f}^{(0)}$ and $k_{f}^{(1)}$ being defined by (35) and 45, respectively.

Figures 1.6 illustrate how the coefficient $k_{f}^{*}$ varies in dependence on the dimensionless forward activation energy $\gamma_{1}^{*}$. Left frames of these figures show the behaviour of the coefficient $k_{f}^{*}$ for $\beta=1$, when we consider the full SRS model with its correction terms (solid lines), and when we remove the correction term from the SRS model (lines with marks $\times \times \times$ ). Right frames of the same figures show how the coefficient $k_{f}^{*}$ is affected when we vary the parameter $\beta$ and consider different proportions of reactive collisions. All curves refer to the full SRS model with its correction term. In particular, the solid lines correspond to $\beta=1$ and is exactly the same one considered in left frames. Besides such curve, we also consider the curves for $\beta=0.75$ (with marks $\times \times \times$ ) and $\beta=0.5$ (with marks $\diamond \diamond \diamond$ ).

Figs. 1, 2 and 3 refer to mixtures $\mathcal{M}_{4}, \mathcal{M}_{10}$ and $\mathcal{M}_{12}$, respectively, when there is an excess of reactants $\left(x_{1}=x_{2}=0.40\right)$ with respect to the products $\left(x_{3}=x_{4}=0.10\right)$ of the forward reaction, and the endothermic reaction dominantes the chemical transformation.

Figs. 4, 5 and 6 refer to mixtures $\mathcal{M}_{3}, \mathcal{M}_{9}$ and $\mathcal{M}_{11}$, respectively, when the reactants are scarce $\left(x_{1}=x_{2}=0.10\right)$ with respect to the products $\left(x_{3}=x_{4}=0.40\right)$ of the forward reaction, and the exothermic reaction is predominant.

\section{(a) Results on the non-equilibrium contributions.}

Below, we provide our comments on the non-equilibrium effects on the coefficient $k_{f}^{(1)}$, when we vary the molecular masses $m_{i}$ and equilibrium concentrations $x_{i}$.

- First, we see that the coefficient $k_{f}^{*}$ is negative, meaning that the first-order rate constant $k_{f}^{(1)}$ is negative, since the zero-order rate constant $k_{f}^{(0)}$ is positive. Furthermore, since $\left|k_{f}^{*}\right|<1$, we 
also see that the non-equilibrium effects induced by the chemical reaction contribute to decrease the absolute value of $k_{f}^{(1)}$ with respect to that of $k_{f}^{(0)}$. Similar results have been obtained in paper [23] for a reactive binary mixture.

- We also see that the non-equilibrium effects induced by the chemical reaction are more noticeable for small values of the activation energy, say $\gamma_{1} \leq 8$, and start to be imperceptible when $\gamma_{1}$ approaches the value 10 . This is a consequence of the fact that a high value of the activation energy indicates that the threshold of the chemical reaction is to high so that reactive collisions become very rare.

- If we estimate the non-equilibrium effects induced by the chemical reaction on the rate constant by the maximum of the absolute value of $k_{f}^{*}$, left diagrams of Figs. 1, 2 , 3 suggest the estimations 0.027 for $\mathcal{M}_{4}, 0.030$ for $\mathcal{M}_{10}$ and 0.033 for $\mathcal{M}_{12}$. Thus, if the endothermic reaction is predominant, we conclude that the non-equilibrium effects become more evident when the reaction heat $E_{a b s} /(k T)$ decreases and the endothermicity character of the forward chemical reaction attenuates, see the last column of Table 5.1.

Moreover, left diagrams of Figs. 4, 5, 6 lead to the estimations 0.032 for $\mathcal{M}_{3}, 0.022$ for $\mathcal{M}_{9}$ and 0.0175 for $\mathcal{M}_{11}$. Thus, if the exothermic reaction is predominant, we conclude that the non-equilibrium effects are more noticeable when the reaction heat $E_{a b s} /(k T)$ is higher and its absolute value is lower so that the exothermicity character of the forward reaction attenuates, see the last column of Table 5.1 .

These results are shown in Fig. 7), where the rate coefficient $k_{f}^{*}$ is displayed in the same frame for the mixtures $\mathcal{M}_{4}, \mathcal{M}_{10}, \mathcal{M}_{12}$ (left diagram) and for the mixtures $\mathcal{M}_{3}, \mathcal{M}_{9}, \mathcal{M}_{11}$ (right diagram).

(b) Results about the impact of the correction terms.

Next, we provide our comments on the effects of the elastic correction terms incorporated in our SRS model.

- When the endothermic reaction is predominant (mixtures $\mathcal{M}_{2}, \mathcal{M}_{4}, \mathcal{M}_{6}, \mathcal{M}_{8}, \mathcal{M}_{10}, \mathcal{M}_{12}$ ), the effects of the correction terms are visible when the masses $m_{3}$ and $m_{4}$ become much more disparate. Therefore, we include only the figures for mixtures $\mathcal{M}_{4}, \mathcal{M}_{10}$ and $\mathcal{M}_{12}$.

On the other hand, when the exothermic chemical reaction is predominant, the effects on $k_{f}^{*}$ are noticeable in all cases considered here (mixtures $\mathcal{M}_{1}, \mathcal{M}_{3}, \mathcal{M}_{5}, \mathcal{M}_{7}, \mathcal{M}_{9}, \mathcal{M}_{11}$ ), but become more relevant when the masses of $m_{3}$ and $m_{4}$ become more disparate, say for mixtures $\mathcal{M}_{9}$ and $\mathcal{M}_{11}$. To show these effects, we include only the figures for mixtures $\mathcal{M}_{3}, \mathcal{M}_{9}$ and $\mathcal{M}_{11}$.

- The impact of the correction terms on the coefficient $k_{f}^{*}$ is recognizable only for small values of the activation energy, say $\gamma_{1}$ around 2 or 3 . This is expected because the correction term is defined through the integration over the sphere $\mathbb{S}_{+}^{2}$ only for those colliding pairs with their kinetic energy exceeding the activation energy (see expression (5)). Therefore, when the activation energy is high, the number of reactive collisions become very small, so that the integral turns out to have a less influence and the double counting becomes less important.

- Left diagrams of Figs. 16 show the impact of the correction terms on $k_{f}^{*}$. We see that, if the endothermic reaction is predominant (Figs. 1 3 ), the correction terms contribute to decrease the absolute value of $k_{f}^{*}$. The maximum deviation is obtained for the mixture $\mathcal{M}_{12}$ and can be estimated to be $5,7 \%$, when measured with respect to the non-equilibrium contribution resulting 
from the full SRS model. This estimation is obtained via the expression $(0.035-0.033) / 0.033$, see Fig. 3 .

- On the other hand, when the exothermic reaction predominates (Figs. 46 6), the correction terms contribute to increase the absolute value of $k_{f}^{*}$. The maximum deviation is obtained for $\mathcal{M}_{11}$ and can be estimated via the expression $(0.0175-0.0135) / 0.0175$ that is $22,9 \%$, see Fig. 6 .

(c) Results about the influence of the parameter $\beta$.

Finally, we provide the comments about the influence of reduction the parameter $\beta$ in different configurations of the full SRS model.

- As expected, smaller values of $\beta$ cause a significant decrease in $\left|k_{f}^{*}\right|$, since reactive collisions become less and less frequent.

- If the endothermic reaction is predominant, right frames of Figs. 1, 2 , 3 show that when $\beta$ decreases from 1 to 0.5 , the reduction observed in $\left|k_{f}^{*}\right|$ is of order $40.7 \%$ for $\mathcal{M}_{4}, 43.3 \%$ for $\mathcal{M}_{10}$ and $44.1 \%$ for $\mathcal{M}_{12}$.

- On the other hand, if the exothermic reaction predominates, right frames of Figs. 4, 5, 6 , show that when $\beta$ decreases from 1 to 0.5 , the reduction observed in $\left|k_{f}^{*}\right|$ is even more relevant being, in particular, of order $48.4 \%$ for $\mathcal{M}_{3}, 50 \%$ for $\mathcal{M}_{9}$ and $54.3 \%$ for $\mathcal{M}_{11}$.

\section{II) Results about the viscosity coefficient.}

In order to investigate the influence of the chemical reaction on the shear viscosity coefficient $\mu$, we estimate instead a dimensionless coefficient

$$
\mu^{*}=\mu / \mu_{I}
$$

where $\mu$ is defined in (48) and $\mu_{I}$ is the viscosity coefficient in a quaternary mixture of nonreacting hard spheres with the same composition as the reactive mixture. Moreover, in view of Remark 2.1. the coefficient $\mu_{I}$ can be obtained from the theory developed in Section 4 for the reactive mixture, by turning off the chemical reaction from the SRS model and setting the coefficients (steric factors) $\beta_{i j}$ equal to zero, for all $i, j=1, \ldots, 4$.

Figures 8 811 , show the behaviour of the coefficient $\mu^{*}$ in dependence on the dimensionless forward activation energy $\gamma_{1}^{*}$. As before, left frames show the behaviour of the coefficient $\mu^{*}$ for $\beta=1$, when we consider the full SRS model with its correction term (solid lines), and when we remove the correction term from the SRS model (lines with marks $\times \times \times$ ). On the other hand, right frames show how the coefficient $\mu^{*}$ is affected when we vary the parameter $\beta$ and consider different proportions of reactive collisions. All curves refer to the full SRS model with its correction terms. In particular, solid lines correspond to $\beta=1$ and are exactly the same as those considered in left frames. The lines with marks $\times \times \times$ correspond to $\beta=0.75$ and with $\diamond \diamond \diamond$ correspond to $\beta=0.5$.

Figures 8 and 9 refer to mixtures $\mathcal{M}_{4}$ and $\mathcal{M}_{12}$, respectively, when the endothermic reaction predominates, whereas Figs. 10 and 11 refer to mixtures $\mathcal{M}_{3}$ and $\mathcal{M}_{11}$ when the exothermic reaction is predominant.

Before discussing the results in detail, let us comment about the unexpected result in the viscosity. In all computations performed with the data as in Table 5.1, and in particular from Figs. 8, 11, we observe that $\mu^{*} \geq 1$ when the full SRS model is considered, showing that, in 
the SRS model, the chemical reaction contributes to increase the viscosity coefficient. On the other hand, we also observe that $\mu^{*} \leq 1$ when we remove the elastic correction terms from the model, showing that, in this situation, the chemical reaction contributes to increase the viscosity coefficient.

In previous papers, see for example [23, 27, the authors conclude that the chemical reaction contributes to decrease the viscosity coefficient (and other transport coefficients). This conclusion is in contrast with our results obtained from the SRS model, but is in agreement with our results obtained when we remove the elastic correction terms from the model.

Therefore, our computations reveal an important feature. In our opinion, at least when we are investigating a chemical regime for which reactive collisions and elastic encounters have comparable characteristic times and are treated in equal par, the elastic correction terms have an important role and should be considered in the modelling equations, in order to avoid double counting of contributions and assure the consistency of the collisional dynamics. In our opinion, the double counting of the collisions hides, in some sense, the effects of the chemical reaction on the transport coefficients.

Our conclusions can be corroborated by the results presented in paper [13] for the shear viscosity. Even if the reactive mixture is not the same and the results are not represented in the same manner, it is evident from some figures included in [13] that for some choices of the model parameters, the viscosity of the reactive mixture is greater than the one of the inert mixture. See, for example, figures 10 and 11 in that paper and also the relevant parameter $M$ defined at page 367 of the same paper.

As explained before, in part I), the effects induced by the chemical reaction are recognizable for $\gamma_{1} \leq 8$ and become almost insignificant when $\gamma_{1}$ approaches the value 10 and the viscosity of the reactive mixture tends to the one of the inert mixture.

(a) Results on the non-equilibrium contributions.

- When we vary the molecular masses $m_{i}$ and equilibrium concentrations $x_{i}$, the computations have shown that, if the endothermic reaction is predominant, the non-equilibrium effects become more evident when the reaction heat $E_{a b s} /(k T)$ decreases and the endothermicity character of the forward chemical reaction attenuates. The left diagram of Fig. 12 exhibits this behaviour, showing in the same frame the viscosity coefficient $\mu^{*}$ for mixtures $\mathcal{M}_{4}$ and $\mathcal{M}_{12}$. See also Figs. 8 and 9 .

- On the other hand, if the exothermic reaction is predominant, the non-equilibrium effects are more noticeable when the reaction heat $E_{a b s} /(k T)$ decreases and its absolute value increases so that the exothermicity character of the forward reaction becomes more significant. This can be seen in the right diagram of Fig. 12, where the viscosity coefficient $\mu^{*}$ is displayed in the same frame for mixtures $\mathcal{M}_{3}$ and $\mathcal{M}_{11}$. See also Figs. 10 and 11 .

- If we estimate the non-equilibrium effects induced by the chemical reaction on the viscosity coefficient by the maximum deviation of $\mu^{*}$ with respect to its reference inert value 1 , left diagrams of Figs. 8 and 9 (endothermic reaction) allow to obtain the estimations 1.19 for $\mathcal{M}_{4}$, and 1.20 for $\mathcal{M}_{12}$, whereas left diagrams of Figs. 10 and 11 (exothermic reaction) lead to the estimations 1.67 for $\mathcal{M}_{3}$, and 2.05 for $\mathcal{M}_{11}$. 
(b) Results about the impact of the correction terms.

- In contrast with what happens to the forward rate constant, see part I), our computations have shown that the correction terms have a great impact on the viscosity coefficient of the reactive mixture. As we have commented above, these terms modify the qualitative behaviour of the viscosity coefficient, in the sense that $\mu^{*}>1$, when the SRS model is considered with its correction terms, whereas $\mu^{*}<1$, when the correction terms are removed from the model.

In our opinion, the correction terms have a relevant effect on the viscosity coefficient because the evaluation of such coefficient directly involves the integration of the reactive and elastic collision operators, see Eqs. (46 48). This is not the case of the forward rate constant, see part I), for which the "main agent" is the reactive operator, see Eqs. 414.45.

- Moreover, from the computations, we have seen that the effects of the correction terms are less significant if the endothermic reaction predominates, and more significant if the exothermic reaction predominates. This can be seen by comparing Figs. 10 and 8 for mixtures $\mathcal{M}_{3}$ and $\mathcal{M}_{4}$, respectively, or Figs. 11 and 9 for mixtures $\mathcal{M}_{11}$ and $\mathcal{M}_{12}$, respectively. Additionally, the effects become even more relevant when the masses $m_{3}$ and $m_{4}$ become much more disparate, as it can be seen by comparing Figs. 10 and 11 or Figs. 8 and 9 .

- The maximum deviation induced by the correction terms, when the endothermic reaction is predominant, for mixture $\mathcal{M}_{12}$ is $21.6 \%$ and can be estimated through the expression $(1.2-$ $0.941) / 1.2$. When the exothermic reaction is predominant, the maximum deviation for mixture $\mathcal{M}_{11}$ is $51.2 \%$, and can be estimated through the expression $(2.05-1) / 2.05$.

(c) Results about the influence of the parameter $\beta$.

The right frames of Figs. 10, 9 depict the influence of parameter $\beta$ in different configurations of the full SRS model.

- From all figures we can see that a reduction in $\beta$ causes a decrease in $\mu^{*}$, indicating that the reactive viscosity coefficient $\mu$ approaches the inert coefficient $\mu_{I}$, when the reactive collisions become less and less frequent.

- If the endothermic reaction is predominant, right frames of Figs. 8 , 9 show that when $\beta$ decreases from 1 to 0.5 , the reduction observed in $\mu^{*}$ is of order $5 \%$ for $\mathcal{M}_{4}$ and $4 \%$ for $\mathcal{M}_{12}$.

- On the other hand, when the exothermic reaction predominates, right frames of Figs. 10, 11 show that when $\beta$ decreases from 1 to 0.5 , the reduction observed in $\left|\mu^{*}\right|$ is even more relevant, being in the order of $18 \%$ for $\mathcal{M}_{3}$ and $32 \%$ for $\mathcal{M}_{11}$.

\section{$6 \quad$ Summary and final remarks}

In this paper we have considered a dilute chemically reactive mixture of four monatomic gases described by the Simple Reactive Spheres (SRS) kinetic model. Using the Chapman-Enskog method, we have determined the second approximate solution to the SRS kinetic equations in a chemical regime for which both elastic and reactive collisions occur with comparable characteristic times. Using the approximate solution, we have evaluated the forward reaction rate coefficient and the shear viscosity coefficient of the reactive mixture. Then, we have investigated two major problems, namely

(a) the influence of the chemical reaction on the two coefficients referred above, and 
(b) the impact of the elastic correction terms, proper of the SRS model, on both coefficients.

Finally, we have also investigated the influence of the probability coefficient $\beta$ of the SRS model on both coefficients.

Summary of the results for the reaction rate coefficient.

- The results for problem (a) with reference to the reaction rate coefficient have shown that the non-equilibrium effects induced by the chemical reaction are more noticeable for small values of the activation energy, as expected. Moreover, such effects become more recognizable when the exothermic reaction is predominant.

- The results for problem (b) with reference to the same coefficient indicate that the elastic correction terms have modest effects. This is a consequence of the fact that this coefficient is strictly related to the reactive operator and has a minor influence of the elastic operator, see Eqs. 41 45 .

We have also observed that the influence of the correction terms is more significant when the molecular masses of the products of the forward reaction become more disparate, indicating that the exothermicity (respectively endothermicity) character increases (respectively attenuates).

Summary of the results for the shear viscosity coefficient.

- An important conclusion that follows from our computations is that, at least in the considered chemical regime in which elastic and reactive collisions have comparable characteristic times, the elastic correction terms have a remarkable influence on the viscosity coefficient. In fact, we have observed that in contrast to the previous works [23, 27], the chemical reaction in the SRS model contributes to increase the viscosity coefficient. This is a consequence of the elastic correction terms, because when we remove these terms from the model dynamics, our results agree with those of papers [23, 27], showing a decreasing of the viscosity coefficient in the reactive mixture.

- With respect to the viscosity coefficient, our computations have shown that the nonequilibrium effects induced by the chemical reaction are more noticeable for small values of the activation energy, as expected. The effects are more noticeable when the exothermic reaction is predominant and, in addition, become greater when the exothermicity (respectively endothermicity) character increases (respectively decreases).

- The results for problem (b) with reference to the viscosity coefficient, our results indicate that the elastic correction terms have a great impact on the viscosity of the reactive mixture, and this is a consequence of the fact that the viscosity coefficient is strictly affected by all collisional events and are particularly dependent on the elastic operators, see Eqs. 46 48). Again, the effects on the viscosity are more significant when the exothermic reaction predominates and become greater when the exothermicity (respectively endothermicity) character increases (respectively decreases).

Finally, the influence of the probability coefficient $\beta$, we can be summarized as follows: a reduction of $\beta$ implies a tendency of the reactive mixture to approximate a non-reactive configuration and, consequently, the reaction rate coefficient decreases significantly and approaches 
zero, whereas the reactive viscosity coefficient approaches the corresponding inert coefficient computed for a non-reactive mixture with the same composition.

We have three final remarks regarding our analysis developed in this paper.

First, our analysis represents a theoretical study in the sense that we refer to an abstract mixture and not to a concrete mixture. On the other hand, experimental data for transport coefficients in reactive mixtures are not available in literature. Therefore we were not able to compare our results with real observations. This perhaps is a limitation of our study. However, starting from this first analysis, we believe that other modelling and computational studies can be developed and some comparisons can be implemented when experimental data is available.

Second, our study shows the importance of the elastic correction term in the collisional dynamics of a reactive mixture, at least when reactive and elastic collisions have comparable characteristic times. The correction terms avoid double counting of collisional contributions and assure the consistency of the model.

Finally, the parametric study developed here offers some guidelines for future investigations and two projects are planed as a continuation of the present work. The first one will deal with the evaluation of the diffusion and thermal conductivity transport coefficients, while in the second, more difficult project, we will study the transport coefficients in a dense regime.

\section{Acknowledgments.}

The paper is partially supported by the Portuguese Funds FCT Project UID/MAT/00013/2013.

\section{References}

[1] R. D. Levine, Molecular Reaction Dynamics, Cambridge University Press, 2005.

[2] V. Giovangigli, Multicomponent flow modeling, Birkhäuser, Boston USA 1999.

[3] E. Nagnibeda and E. Kustova, Non-Equilibrium Reacting Gas Flows - Kinetic Theory of Transport and Relaxation Processes, Springer, Berlin, 2009.

[4] G. M. Kremer, An introduction to the Boltzmann equation and transport processes in gases, Springer, Berlin, 2010.

[5] I. Prigogine and E. Xhrouet, "On the perturbation of maxwell distribution function by chemical reactions in gases", Physica, 15, 913-932 (1949).

[6] I. Prigogine and M. Mahieu, "Sur la perturbation de la distribution de Maxwell par des réactions chimiques en phase gazeuse", Physica, 16, 51-64 (1950).

[7] R. D. Present, "On the velocity distribution in a chemically reacting gas", J. Chem. Phys., 31, 747-750 (1959).

[8] R. D. Present, "Chapman-Enskog method in chemical kinetics", J. Chem. Phys., 48, 48754877 (1968). 
[9] G. Ludwig and M. Heil, "Boundary-layer theory with dissociation and ionization", Advances in Applied Mechanics, 6, 39-118 (1960).

[10] J. Ross and P. Mazur, "Some deductions from a formal statistical mechanical theory of chemical kinetics", J. Chem. Phys., 35, 19-28 (1961).

[11] B. Shizgal and M. Karplus, "Nonequilibrium contributions to the rate of reaction. I. Perturbation of the velocity distribution function", J. Chem. Phys., 52, 4262-4278 (1970).

[12] B. Shizgal and M. Karplus, "Nonequilibrium contributions to the rate of reactions II. Isolated multicomponent systems", J. Chem. Phys., 54, 4345-4356 (1971).

[13] N. Xystris and J. S. Dahler, "Mass and momentum transport in dilute reacting gases", J. Chem. Phys., 68, 354-373 (1978).

[14] A. S. Cukrowski and J. Popielawski, "The effect of viscous flow and thermal flux on the rate of chemical reaction in dilute gases", Chem. Phys., 109, 215-226 (1986).

[15] A. S. Cukrowski, J. Popielawski, L. Qin and J. S. Dahler, "A simplified theoretical analysis of nonequilibrium effects in bimolecular gas phase reactions", J. Chem. Phys., 97, 9086-9093 (1992).

[16] B. V. Alexeev, A. Chikhaoui and I. T. Grushin, "Application of the generalized ChapmanEnskog method to the transport-coefficient calculation in a reacting gas mixture", Phys. Rev. E, 49, 2809-2825 (1994).

[17] B. Shizgal and D. Napier "Nonequilibrium effects in reactive systems; the effect of reaction products and the validity of the Chapman-Enskog method", Physica A, 223, 50-86 (1996).

[18] A. Ern and V. Giovangigli, "The kinetic chemical equilibrium regime", Physica A, 260, 49-72 (1998).

[19] E. Kustova and E.Nagnibeda, "Transport properties of a reacting gas mixture with strong vibrational and chemical nonequilibrium", Chem. Phys., 233, 57-75 (1998).

[20] A. Rossani and G. Spiga, "A note on the kinetic theory of chemically reacting gases", Physica A, 272, 563-573 (1999).

[21] M. Groppi and G. Spiga, "Kinetic approach to chemical reactions and inelastic transitions in a rarefied gas", J. Math. Chem., 26, 197-219 (1999).

[22] A. S. Cukrowski, "The role of products and a reverse reaction in analysis of nonequilibrium effects in a bimolecular chemical reaction in a dilute gas", Physica A, 275, 134-151 (2000).

[23] G. M. Alves and G. M. Kremer, "Effect of chemical reactions on the transport coefficients of binary mixtures", J. Chem Phys., 117, 2205-2215 (2002).

[24] A. Ern and V. Giovangigli, "Kinetic theory of reactive gas mixtures with application to combustion", Transp. Theor. Stat. Phys., 32, 657-677 (2003).

[25] V. Giovangigli and B. Graille, "Kinetic theory of partially ionized reactive gas mixtures", Physica A, 327, 313-348 (2003). 
[26] L. Desvillettes, R. Monaco and F. Salvarani, "A kinetic model allowing to obtain the energy law of polytropic gases in the presence of chemical reactions", Eur. J. Mech. B Fluids, 24, 219-236 (2005).

[27] A. W. Silva, G. M. Alves and G. M. Kremer, "Transport phenomena in a reactive quaternary gas mixture", Physica A, 374, 533-548 (2007).

[28] M. Groppi and G. Spiga, "A Kinetic relaxation model for bimolecular chemical reactions", Bulletin of the Institute of Mathematics Academia Sinica, 2, 609-635 (2007).

[29] G. M. Kremer and A. J. Soares, "Effect of reaction heat on Maxwellian distribution functions and rate of reactions", J. Stat. Mech. P12003, 1-16 (2007).

[30] E. Kustova and G. M. Kremer, "Chemical reaction rates and non-equilibrium pressure of reacting gas mixtures in the state-to-state approach", Chem. Phys., 445, 82-94 (2014).

[31] L. Qin and J. S. Dahler, "The kinetic theory of a simple, chemically reactive fluid: scattering functions and relaxation processes", J. Chem. Physics, 103, 725-750 (1995).

[32] J. S. Dahler and L. Qin, "Nonequilibrium statistical mechanics of chemically reactive fluids", J. Chem. Physics, 118, 8396-8404 (2003).

[33] J. Polewczak, "The kinetic theory of simple reacting spheres: I. Global existence result in a dilute-gas case", J. Stat. Physics, 100, 327-362 (2000).

[34] M. Groppi and J. Polewczak, "On two kinetic models for chemical reactions: comparisons and existence results", J. Stat. Physics, 117, 211-241 (2004).

[35] J. Polewczak and A. J. Soares, "Kinetic Theory of Simple Reacting Spheres I", 27th International Symposium on Rarefied Gas Dynamics, 2010, Pacific Grove, AIP Conference Proceedings, Vol. 1333, pp. 117-122 (2011).

[36] F. Carvalho, J. Polewczak and A. J. Soares, "On the kinetic systems for simple reacting spheres: modeling and linearized equations", Particle Systems and Partial Differential Equations, 2012, Braga, Springer Proceedings in Mathematics \& Statistics, Vol. 75, pp. 251-267 (2014).

[37] J. Polewczak and A. J. Soares, "On modified simple reacting spheres kinetic model for chemically reactive gases", Kinetic and Related Models, 10, 513-539 (2017).

[38] S. Chapman and T. G. Cowling, The mathematical theory of non-uniform gases, Cambridge University Press, Cambridge 1970.

[39] J. Ferziger and H. Kaper, Mathematical Theory of Transport Processes in Gases, NorthHolland, Amsterdam 1972.

[40] W. Fickett, Introduction to Detonation Theory, Berkeley, University of California, 1986.

[41] C. Cercignani, The Boltzmann Equation and its Applications, Springer, New York, 1988. 

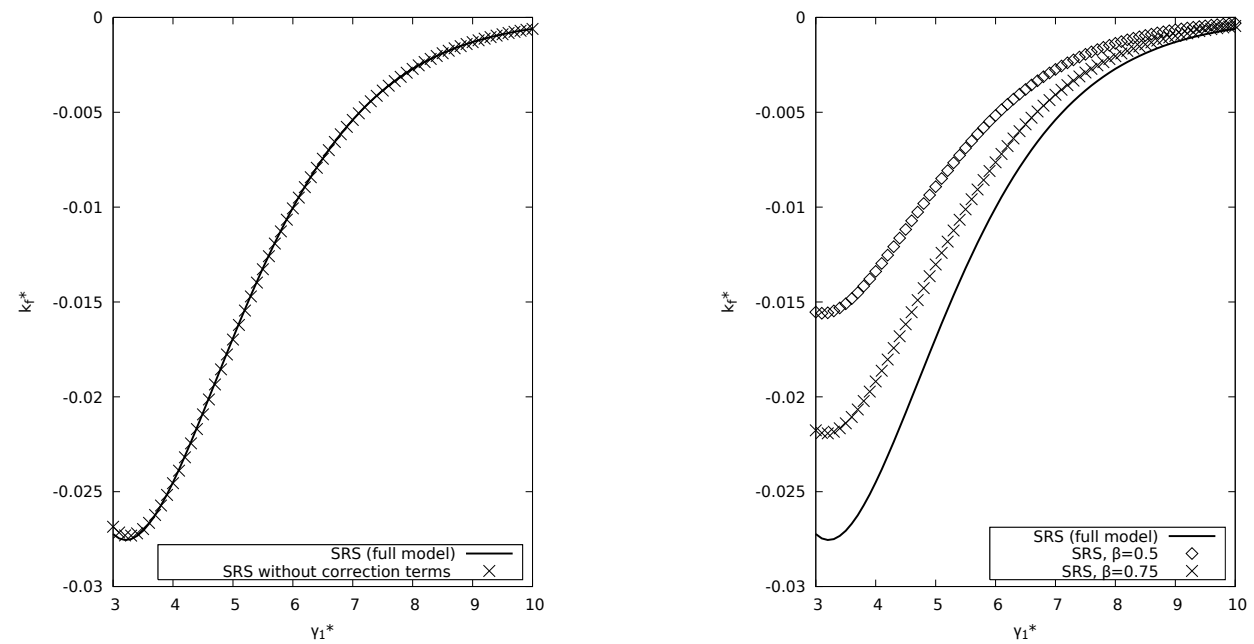

Figure 1: Mixture $\mathcal{M}_{4}$ (endothermic forward reaction, $E_{a b s}=2.634, \mu_{34} / \mu_{12}=0.758$ ). Rate constant of the forward reaction, $k_{f}^{*}$, versus the forward activation energy $\gamma_{1}^{*}$. Left frame: Effects of the correction terms. Full SRS model and SRS model without correction terms. Right frame: Effects of the parameter $\beta$. Full SRS model with $\beta=1, \beta=0.75$ and $\beta=0.5$.
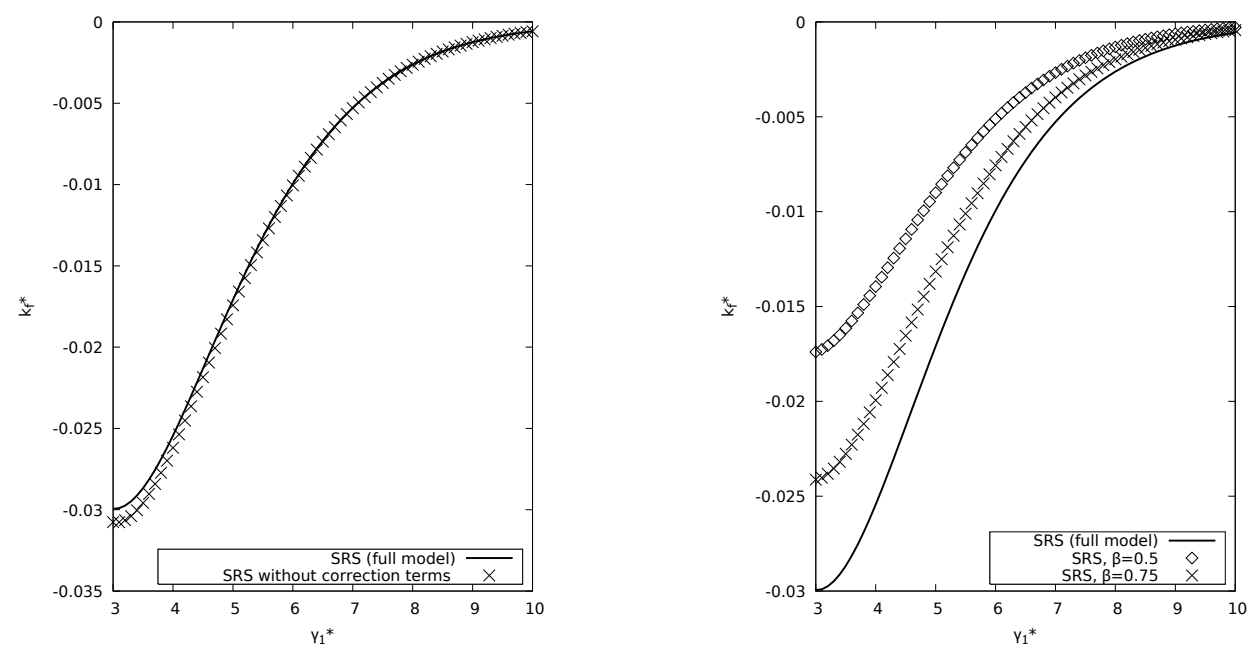

Figure 2: Mixture $\mathcal{M}_{10}$ (endothermic forward reaction, $E_{a b s}=2.267, \mu_{34} / \mu_{12}=0.364$ ). Rate constant of the forward reaction, $k_{f}^{*}$, versus the forward activation energy $\gamma_{1}^{*}$. Left frame: Effects of the correction terms. Full SRS model and SRS model without correction terms. Right frame: Effects of the parameter $\beta$. Full SRS model with $\beta=1, \beta=0.75$ and $\beta=0.5$. 

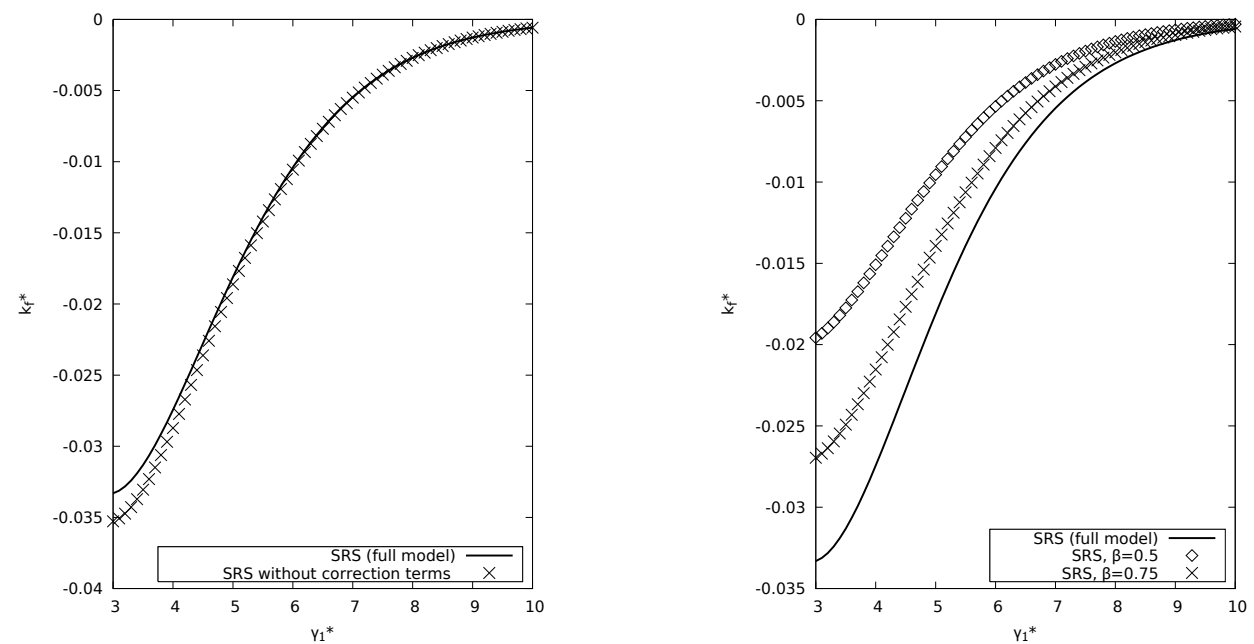

Figure 3: Mixture $\mathcal{M}_{12}$ (endothermic forward reaction, $E_{a b s}=1.947, \mu_{34} / \mu_{12}=0.192$ ). Rate constant of the forward reaction, $k_{f}^{*}$, versus the forward activation energy $\gamma_{1}^{*}$. Left frame: Effects of the correction terms. Full SRS model and SRS model without correction terms. Right frame: Effects of the parameter $\beta$. Full SRS model with $\beta=1, \beta=0.75$ and $\beta=0.5$.
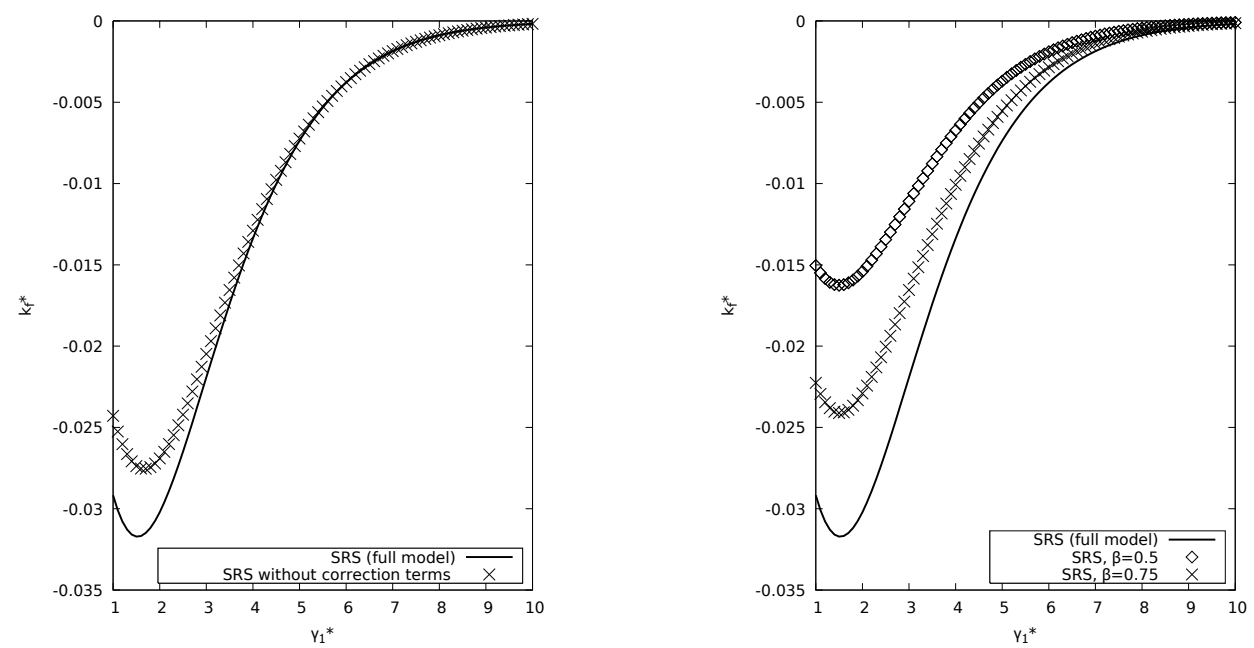

Figure 4: Mixture $\mathcal{M}_{3}$ (exothermic forward reaction, $E_{a b s}=-2.911, \mu_{34} / \mu_{12}=0.758$ ). Rate constant of the forward reaction, $k_{f}^{*}$, versus the forward activation energy $\gamma_{1}^{*}$. Left frame: Effects of the correction terms. Full SRS model and SRS model without correction terms. Right frame: Effects of the parameter $\beta$. Full SRS model with $\beta=1, \beta=0.75$ and $\beta=0.5$. 

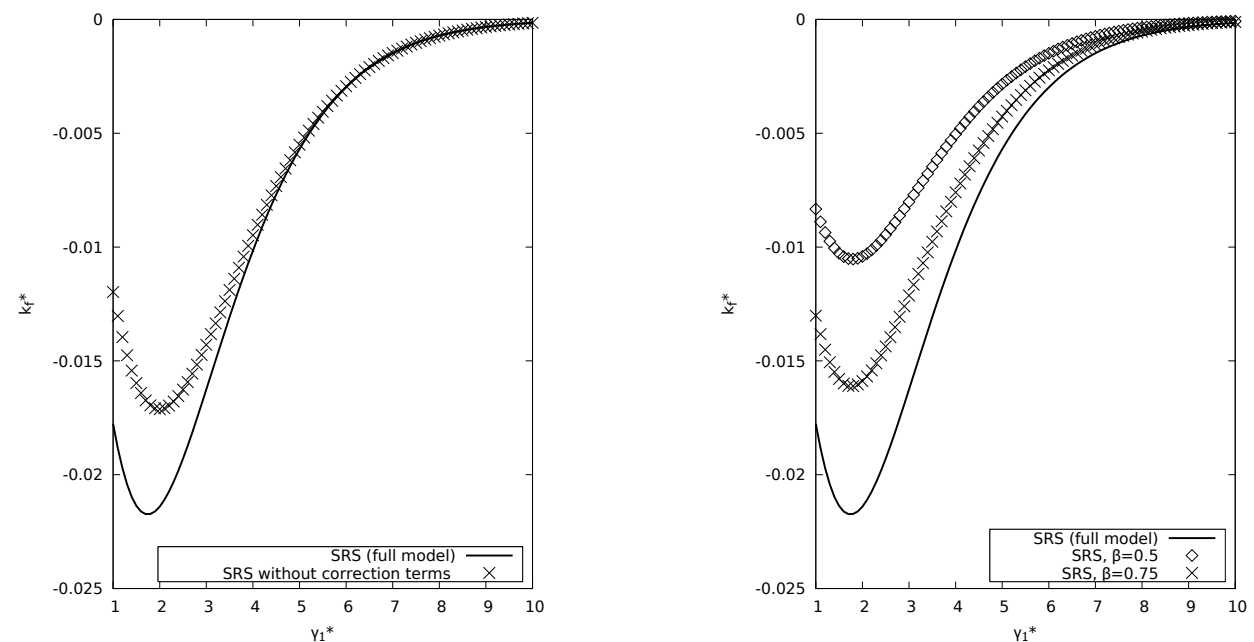

Figure 5: Mixture $\mathcal{M}_{9}$ (exothermic forward reaction, $E_{a b s}=-3.278, \mu_{34} / \mu_{12}=0.364$ ). Rate constant of the forward reaction, $k_{f}^{*}$, versus the forward activation energy $\gamma_{1}^{*}$. Left frame: Effects of the correction terms. Full SRS model and SRS model without correction terms. Right frame: Effects of the parameter $\beta$. Full SRS model with $\beta=1, \beta=0.75$ and $\beta=0.5$.
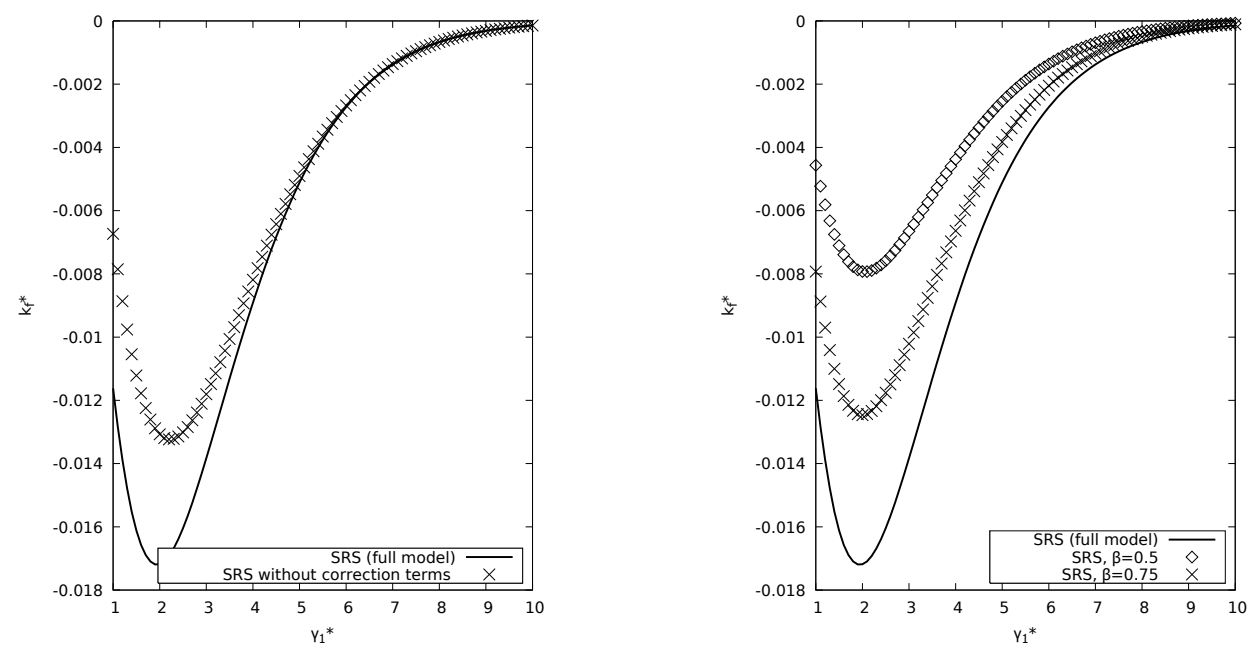

Figure 6: Mixture $\mathcal{M}_{11}$ (exothermic forward reaction, $E_{a b s}=-3.598, \mu_{34} / \mu_{12}=0.192$ ). Rate constant of the forward reaction, $k_{f}^{*}$, versus the forward activation energy $\gamma_{1}^{*}$. Left frame: Effects of the correction terms. Full SRS model and SRS model without correction terms. Right frame: Effects of the parameter $\beta$. Full SRS model with $\beta=1, \beta=0.75$ and $\beta=0.5$. 

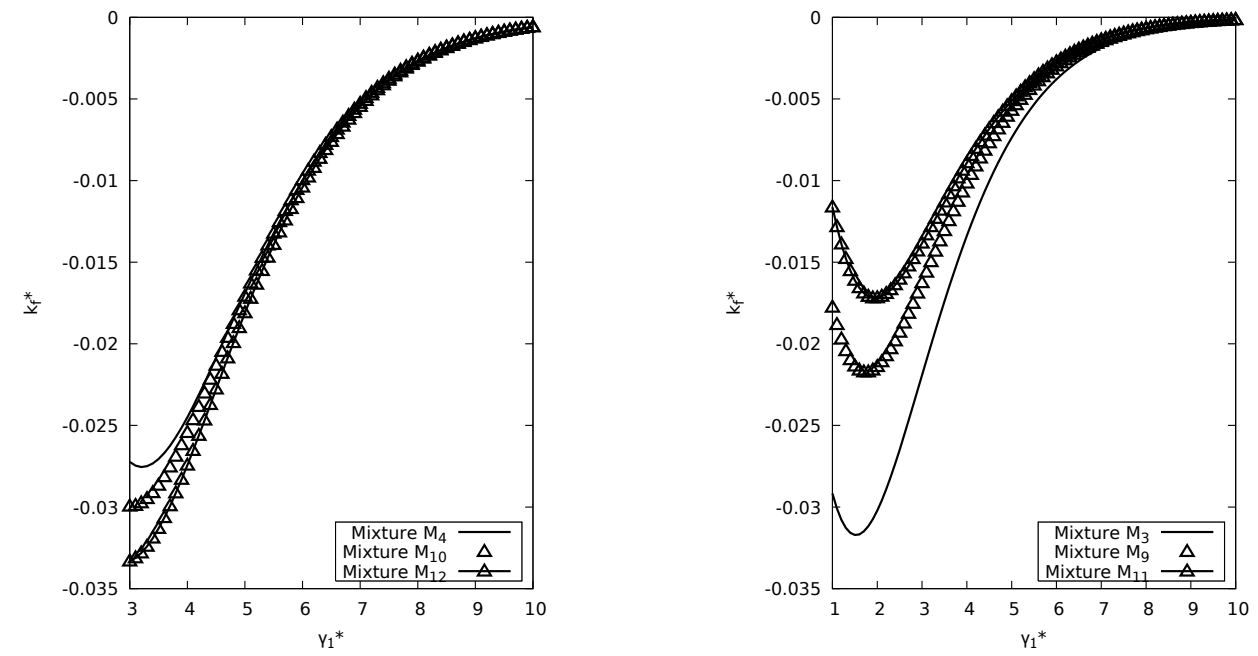

Figure 7: Non-equilibrium effects induced by the chemical reaction on the rate constant $k_{f}^{*}$. Full SRS model, $\beta=1$. Left frame: endothermic forward reaction, mixtures $\mathcal{M}_{4}, \mathcal{M}_{10}$ and $\mathcal{M}_{12}$. Right frame: exothermic forward reaction, mixtures $\mathcal{M}_{3}, \mathcal{M}_{9}$ and $\mathcal{M}_{11}$.
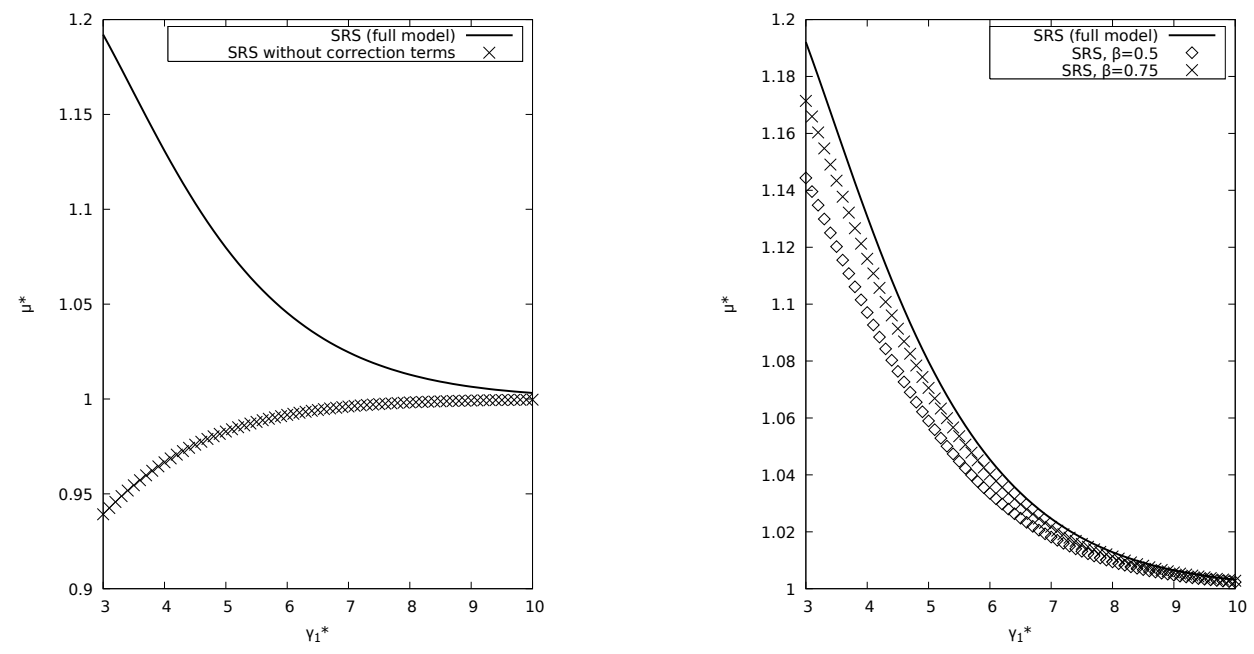

Figure 8: Mixture $\mathcal{M}_{4}$ (endothermic forward reaction, $E_{a b s}=2.634, \mu_{34} / \mu_{12}=0.758$ ). Viscosity, $\mu^{*}$, versus the forward activation energy $\gamma_{1}^{*}$. Left frame: Effects of the correction terms. Full SRS model and SRS model without correction terms. Right frame: Effects of the parameter $\beta$. Full SRS model with $\beta=1, \beta=0.75$ and $\beta=0.5$. 

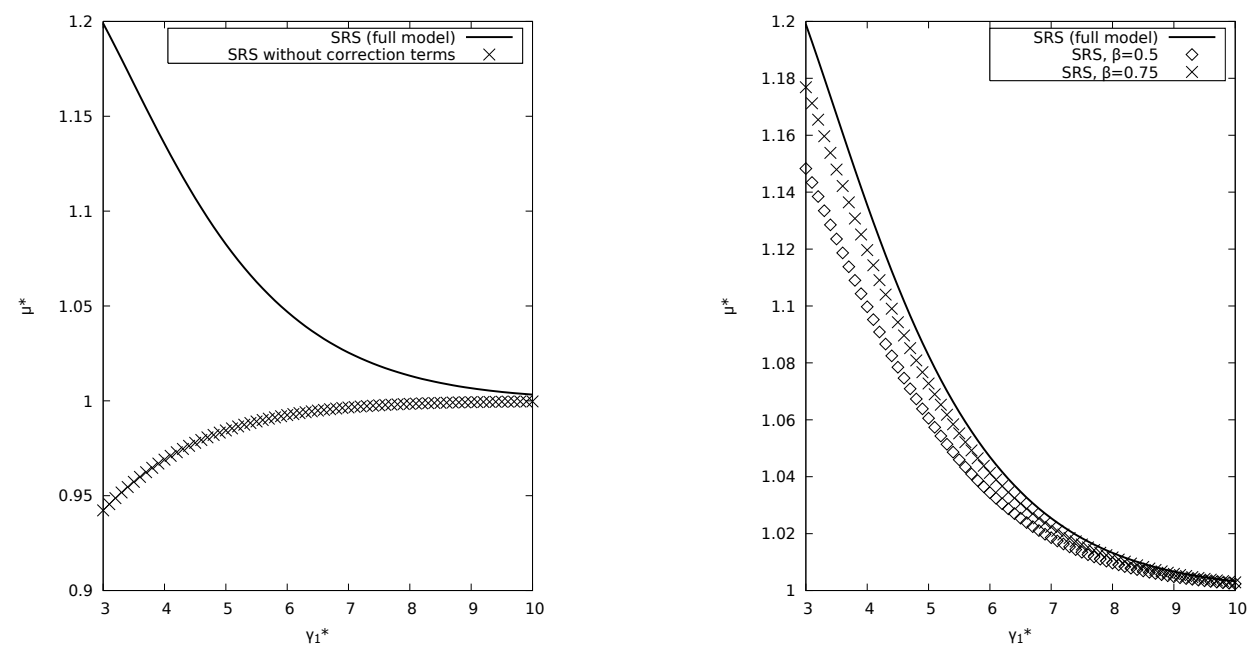

Figure 9: Mixture $\mathcal{M}_{12}$ (endothermic forward reaction, $E_{\text {abs }}=1.947, \mu_{34} / \mu_{12}=0.192$ ). Viscosity, $\mu^{*}$, versus the forward activation energy $\gamma_{1}^{*}$. Left frame: Effects of the correction terms. Full SRS model and SRS model without correction terms. Right frame: Effects of the parameter $\beta$. Full SRS model with $\beta=1, \beta=0.75$ and $\beta=0.5$.
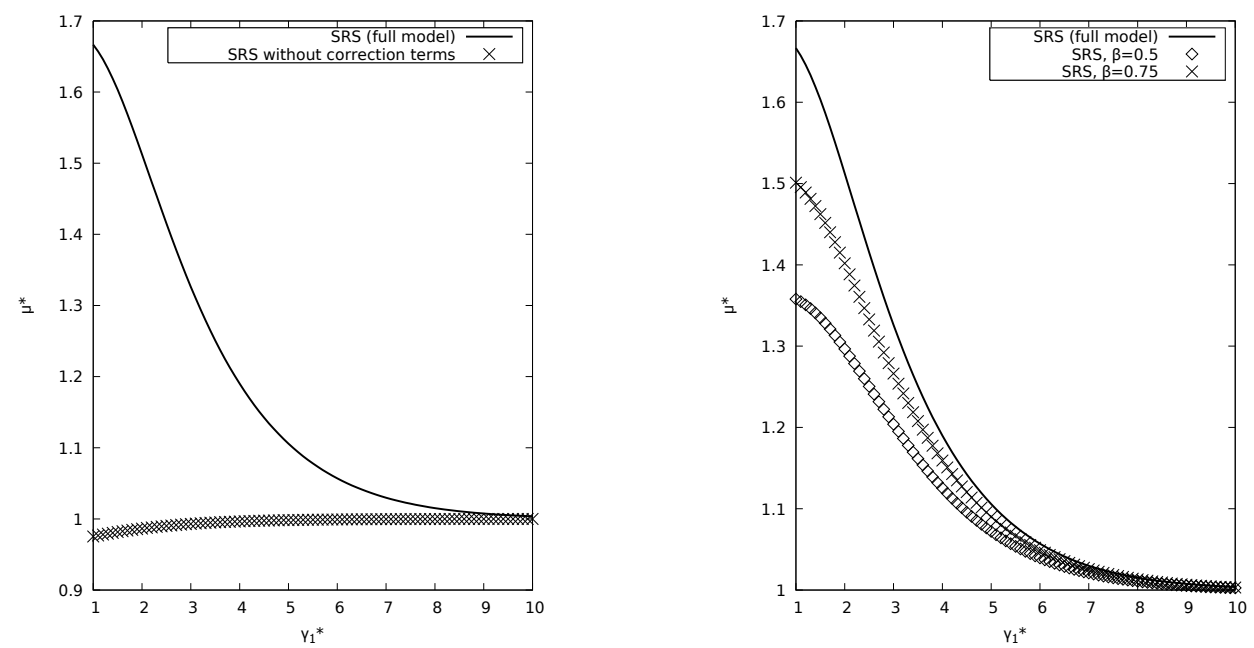

Figure 10: Mixture $\mathcal{M}_{3}$ (exothermic forward reaction, $E_{a b s}=-2.911, \mu_{34} / \mu_{12}=0.758$ ). Viscosity, $\mu^{*}$, versus the forward activation energy $\gamma_{1}^{*}$. Left frame: Effects of the correction terms. Full SRS model and SRS model without correction terms. Right frame: Effects of the parameter $\beta$. Full SRS model with $\beta=1, \beta=0.75$ and $\beta=0.5$. 

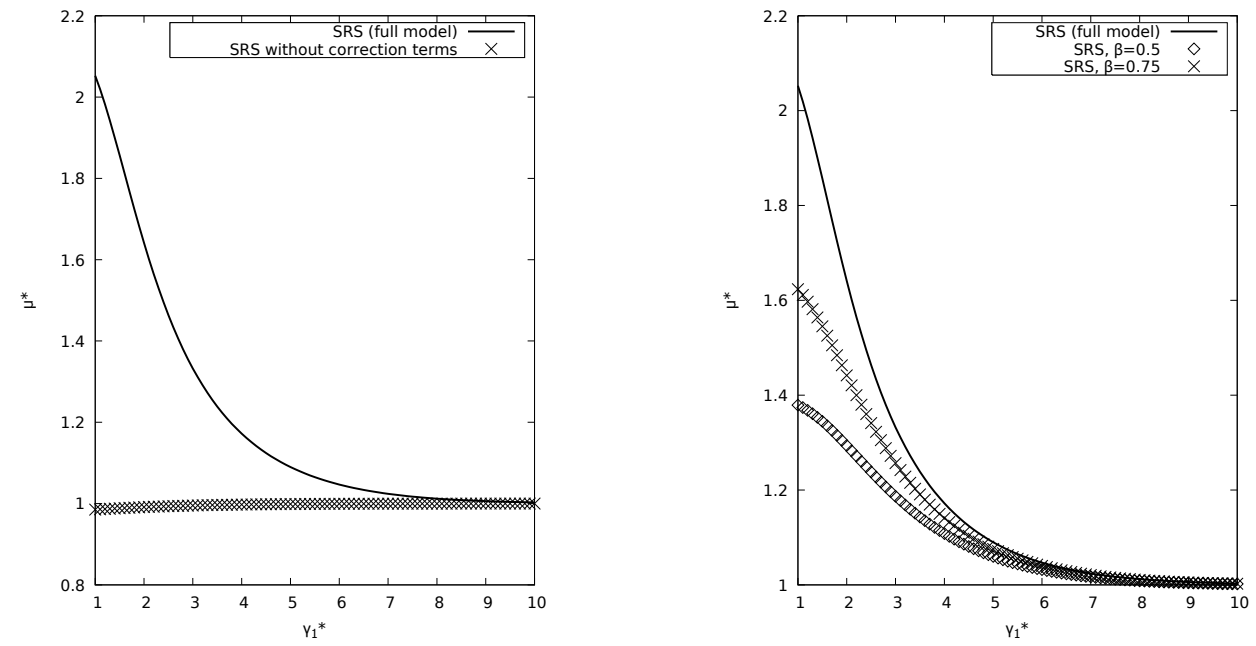

Figure 11: Mixture $\mathcal{M}_{11}$ (exothermic forward reaction, $E_{a b s}=-3.598, \mu_{34} / \mu_{12}=0.192$ ). Viscosity, $\mu^{*}$, versus the forward activation energy $\gamma_{1}^{*}$. Left frame: Effects of the correction terms. Full SRS model and SRS model without correction terms. Right frame: Effects of the parameter $\beta$. Full SRS model with $\beta=1, \beta=0.75$ and $\beta=0.5$.
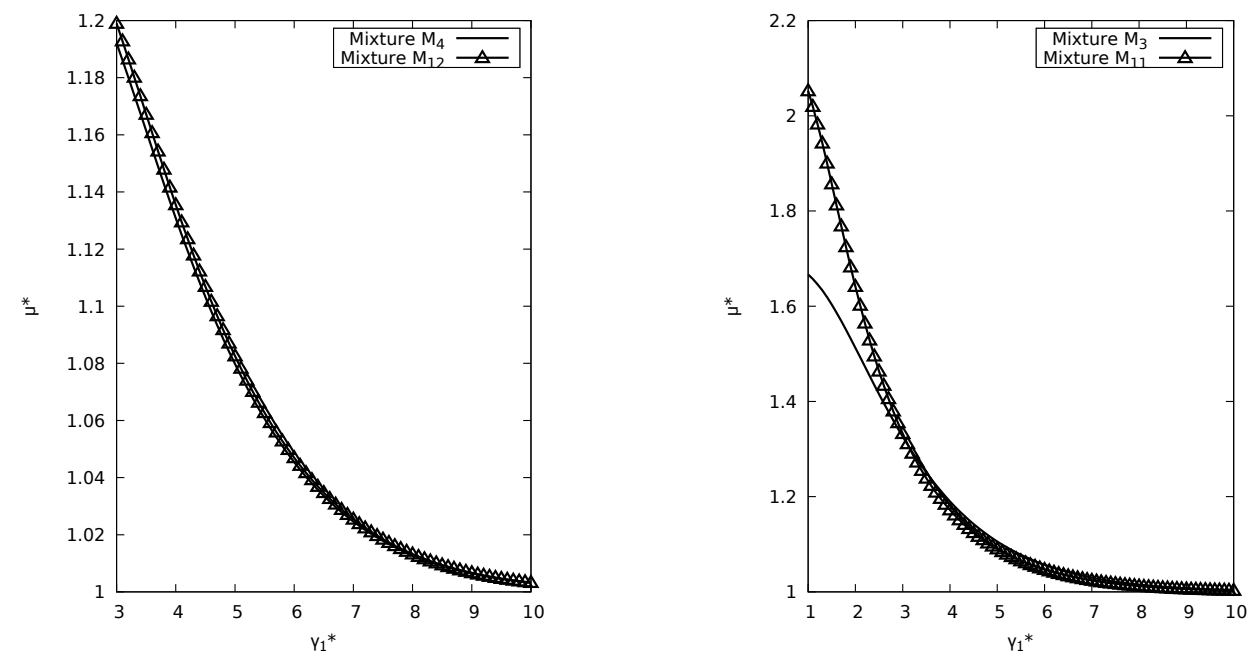

Figure 12: Non-equilibrium effects induced by the chemical reaction on the viscosity coefficient $\mu^{*}$. Full SRS model, $\beta=1$. Left frame: endothermic forward reaction, mixtures $\mathcal{M}_{4}$ and $\mathcal{M}_{12}$. Right frame: exothermic forward reaction, mixtures $\mathcal{M}_{3}$ and $\mathcal{M}_{11}$. 